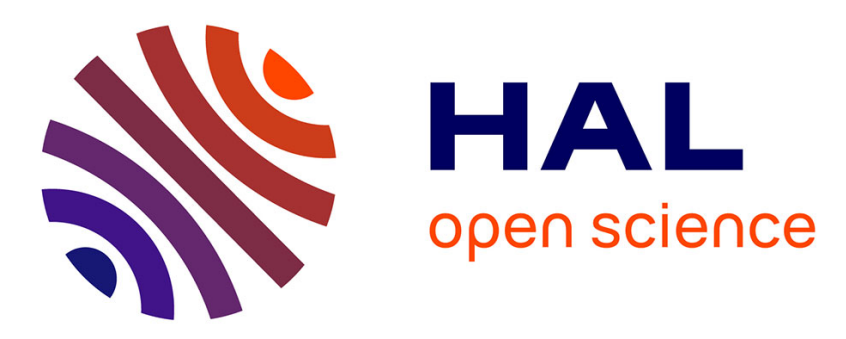

\title{
Analytical shape uncertainties in the polyhedron gravity model
}

Benjamin Bercovici, Paolo Panicucci, Jay Mcmahon

\section{To cite this version:}

Benjamin Bercovici, Paolo Panicucci, Jay Mcmahon. Analytical shape uncertainties in the polyhedron gravity model. Celestial Mechanics and Dynamical Astronomy, 2020, 132 (5), pp.1-32. 10.1007/s10569-020-09967-3 . hal-03033828

\section{HAL Id: hal-03033828 \\ https://hal.science/hal-03033828}

Submitted on 1 Dec 2020

HAL is a multi-disciplinary open access archive for the deposit and dissemination of scientific research documents, whether they are published or not. The documents may come from teaching and research institutions in France or abroad, or from public or private research centers.
L'archive ouverte pluridisciplinaire HAL, est destinée au dépôt et à la diffusion de documents scientifiques de niveau recherche, publiés ou non, émanant des établissements d'enseignement et de recherche français ou étrangers, des laboratoires publics ou privés. 


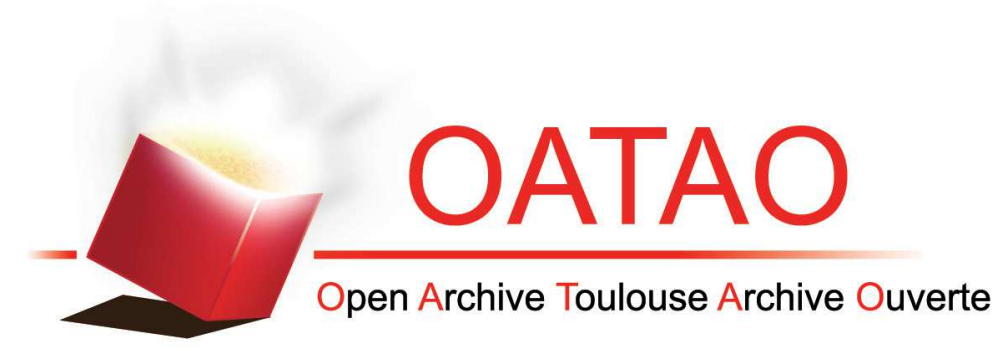

\section{Open Archive Toulouse Archive Ouverte (OATAO)}

OATAO is an open access repository that collects the work of some Toulouse researchers and makes it freely available over the web where possible.

This is an author's version published in: https://oatao.univ-toulouse.fr/26910

Official URL: https://doi.org/10.1007/s10569-020-09967-3

\section{To cite this version :}

Bercovici, Benjamin and Panicucci, Paolo and McMahon, Jay Analytical shape uncertainties in the polyhedron gravity model. (2020) Celestial Mechanics and Dynamical Astronomy, 132 (5). ISSN 0923-2958

Any correspondence concerning this service should be sent to the repository administrator: tech-oatao@listes-diff.inp-toulouse.fr 


\title{
Analytical shape uncertainties in the polyhedron gravity model
}

\author{
Benjamin Bercovici $^{1}$ - Paolo Panicucci ${ }^{2,3,4} \cdot$ Jay McMahon $^{1}$
}

\begin{abstract}
The exploration of small bodies in the Solar system and the ability to perform remote or in situ science is tied to the understanding of the dynamical environment of such objects. As such, the evaluation of the gravity field arising from small bodies is key to this understanding. However, remote observations can only produce shape estimates, from which only uncertain gravity fields can be computed. The current disconnect in the literature between the uncertainty in the shape and that of the gravity field properties is detrimental to small body science and robust mission design. In particular, the literature does not provide any quantitative means to capture this link in the polyhedron gravity model, one of the main gravity model representations. With this in mind, this paper derives the expressions of the first variations and partial derivatives in the potential, acceleration and slopes computed from the polyhedron gravity model with respect to the vertices of the underlying body. These formulae are then combined with a Gaussian description of the uncertainty in the vertex coordinates so as to obtain analytical predictions of the potential, slope variances as well as the covariance in the acceleration at arbitrary locations around the body, treated as a stochastic shape. This linearized analytical approach was able to capture the statistical variation in the dynamical environment about asteroid 25143 Itokawa and 16 Psyche under the assumption of stochastic errors in the bodies' shape models, at a lower computational cost than Monte-Carlo simulations. These methods should be of benefit to planetary scientists and mission designers seeking for more insight into the dynamical environment of uncertain small body shapes.
\end{abstract}

Keywords Polyhedron gravity · Uncertainty quantification - Stochastic shape - Dynamical environment

\footnotetext{
$\triangle$ Benjamin Bercovici

benjamin.bercovici@gmail.com

1 University of Colorado, Boulder, USA

2 Centre National d'Études Spatiales, Toulouse, France

3 ISAE-SUPAERO, Toulouse, France

4 Airbus Defence and Space, Toulouse, France
} 


\section{Introduction and motivation}

The determination of a small body's inertia properties — such as volume, center-of-mass and inertia tensor-as well as that of its gravity field is deeply intertwined with the knowledge of its outer shape. This is why remote observations of small bodies by means of radar or optical telescopes are paramount to gaining such understanding before in situ observations can be collected by dedicated missions (Ostro et al. 2002). Once shape model and mass estimates are available, the dynamical environment of the small body can be assessed. Two major gravity models suitable for small body gravity computations are the polyhedron gravity model (Werner and Scheeres 1997), and the spherical harmonics expansion of the potential arising from polyhedral shapes (Werner 1997). In summary, the PGM is an exact representation of the gravity field of a constant-density polyhedron, obtained from the integration and summation of the potential arising from constant-density tetrahedra, while spherical harmonics expansions are obtained by truncating the infinite series yielding the gravitational potential integrated over each tetrahedron. Due to their complementary nature, both models are used extensively in small body science and mission design, operations (Owen and Wang 2001; Groussin et al. 2015; Williams et al. 2001; Pesce et al. 2018; Broschart et al. 2017).

However, it can be noted that the treatment of shape uncertainty by the small body science and engineering community lacks a formal framework. Previous works on shape reconstruction and dynamical environment characterization of small bodies do acknowledge the presence of uncertainty in the reconstructed shapes and inertia (Scheeres et al. 2006a; Nolan et al. 2013; Scheeres et al. 2016; Busch et al. 2006; Torppa et al. 2003), but do not go as far as establishing the relationship between the uncertainty in the shape and that in the inertia properties and gravity field in a systematic and quantitative way.

Muinonen has proposed the so called Gaussian shape hypothesis, which consisted in decomposing a shape of interest as a spherical expansion of random Gaussian variables (Muinonen 1998) so as to extract its inertia moment statistics. Yet, this approach cannot handle arbitrary body shapes, as well as being not directly related to polyhedron shape models, the workhorse of today's shape models parametrization in the small body astronomy field (Torppa et al. 2008). Uncertainties in the subsequent gravity field are also not investigated. Mass concentrations have been leveraged in the past to describe the uncertainty in the gravity field in proximity to a small body (Ren and Shan 2015), and dedicated MonteCarlo analyses are typically used to investigate the effect of uncertain spherical harmonics coefficients on spacecraft trajectories (Melman et al. 2013). In an attempt to improve upon these sampling-based approaches, recent work by the authors of the present paper has documented the quantitative link between the uncertainty in the shape and that in the inertia parameters (Bercovici and Mcmahon 2019), spherical harmonics representation of the gravity field (Panicucci et al. 2020), by developing the expressions of the first variations in the quantities of interest relative to the shape vertex coordinates, and treating the variation in the vertices coordinates as a Gaussian vector. The second moments about the mean of the resulting Gaussian distributions in the quantities of interest are then readily available. Yet, the lack of formal expressions for the uncertainties in the polyhedral gravity field emanating from an uncertain shape still remains. The present paper aims at filling this gap, by deriving analytical expressions for the partial derivative in the potential and accelerations arising from the polyhedron gravity model of a small body relative to the vertices comprising the shape. Treating the vertex coordinates as a Gaussian vector of known mean and covariance, formal expressions for the sought-for uncertainties in the gravity potential, acceleration and surface slopes can be obtained. 


\section{Notations}

The following notations are used throughout this paper:

- Scalar variables are written in plain font, as in $a$ or $A$.

- Non-unit vectors are denoted as bold quantities such as $\mathbf{n}$ or $\mathbf{N}$. One-dimensional vector quantities, when ambiguous, are wrapped in parentheses, as in $\left(\partial \Omega_{f} / \partial \mathbf{T}_{f}\right)$.

- $\mathbf{0}_{n}$ is the zero column-vector of dimension $n$.

- Unit-vectors are denoted as 'hatted' variables, as in $\hat{u}$.

- Matrices are wrapped in square brackets, as in $\left[F_{f}\right]$.

- $[I]_{n}$ is the identity square-matrix of dimension $n$.

- $[0]_{n, m}$ is the zero matrix of dimensions $n, m$.

- The matrix representation of the left-hand cross-product operator $\mathbf{y} \mapsto \mathbf{x} \times \mathbf{y}$ is written $[\mathbf{x}]^{\times}$.

- $[\mathcal{A B}]$ denotes the orthogonal direction cosine matrix converting vector coordinates from the $\mathcal{B}$ reference frame to the $\mathcal{A}$ reference frame.

- $N_{C}$ refers to the number of vertices in a considered shape.

- $N_{f}$ refers to the number of facets in a considered shape.

- $N_{e}$ refers to the number of edges in a considered shape.

\section{The polyhedron gravity model in brief}

Werner and Scheeres proposed closed form expressions of the potential created by a constantdensity polyhedral shape comprised of triangular surface elements (dubbed "facets"), from which expressions of the gravity acceleration and gravity gradient matrix could be readily derived (Werner and Scheeres 1997). These expressions are known as that of the polyhedron gravity model, or PGM. The present section summarizes the main equations that constitutes the PGM, following the same notations as Werner and Scheeres. Let $\mathbf{C}$ be the vector storing the stacked-up coordinates of all the vertices forming the shape: $\mathbf{C}=\left(\mathbf{C}_{1}^{T}, \ldots, \mathbf{C}_{N_{C}}\right)^{T} \in \mathbb{R}^{3 N_{C}}$. Denote the $i$-th shape vertex coordinates as $\mathbf{C}_{i}\left(i \in\left\{1,2, \ldots, N_{C}\right\}\right)$. Denote the $f$-th facet in the shape by $f\left(f \in\left\{1,2, \ldots, N_{f}\right\}\right)$. Let $i_{f, j}^{F}$ be the facet connectivity table $(j \in\{1,2,3\})$. The $f$-facet is formed by associating in a counter-clockwise fashion the points $\mathbf{C}_{i_{f, 1}^{F}}, \mathbf{C}_{i_{f, 2}^{F}}$ and $\mathbf{C}_{i_{f, 3}^{F}} . i_{f, 1}^{F}, i_{f, 2}^{F}$ and $i_{f, 3}^{F}$ are the global indices of the first, second and third vertices forming the facet indexed by $f$. Denote the $e$-th edge in the shape by $e\left(e \in\left\{1,2, \ldots, N_{e}\right\}\right)$. Let $i_{e, j}^{E}$ be the edge connectivity table $(j \in\{1,2\})$. The $e$-th edge is thus formed by connecting two points $\mathbf{C}_{i_{e, 1}^{E}}$ and $\mathbf{C}_{i_{e, 2}^{E}} . i_{e, 1}^{E}$ and $i_{e, 2}^{E}$ denote the global indices of the first and second point on the edge indexed by $e$, respectively. Finally, let $f_{e, 1}^{E F}, f_{e, 2}^{E F}$ denote the facet indices of the first and second facet whose intersection defines edge $e$. Let $\mathbf{r}$ be the coordinates of the field point where one wishes to compute the PGM quantities of interest, expressed in the same reference frame as the vertex coordinates. The potential, acceleration and gravity-gradient matrix arising from a polyhedron shape comprised of $N_{f}$ facets and $N_{e}$ edges of uniform density $\rho$, evaluated at the field point $\mathbf{r}$, take the form

$$
U(\mathbf{r})=\frac{G \rho}{2}\left[\sum_{e=1}^{N_{e}} \mathbf{r}_{i_{e, 1}^{E}}^{T}\left[E_{e}\right] \mathbf{r}_{i_{e, 1}^{E}} L_{\mathrm{e}}-\sum_{f=1}^{N_{f}} \mathbf{r}_{i_{f, 1}^{F}}^{T}\left[F_{f}\right] \mathbf{r}_{i_{f, 1}^{F}} \Omega_{\mathrm{f}}\right],
$$


Fig. 1 Facet-Edge geometry in definition of edge dyad (Werner and Scheeres 1997)

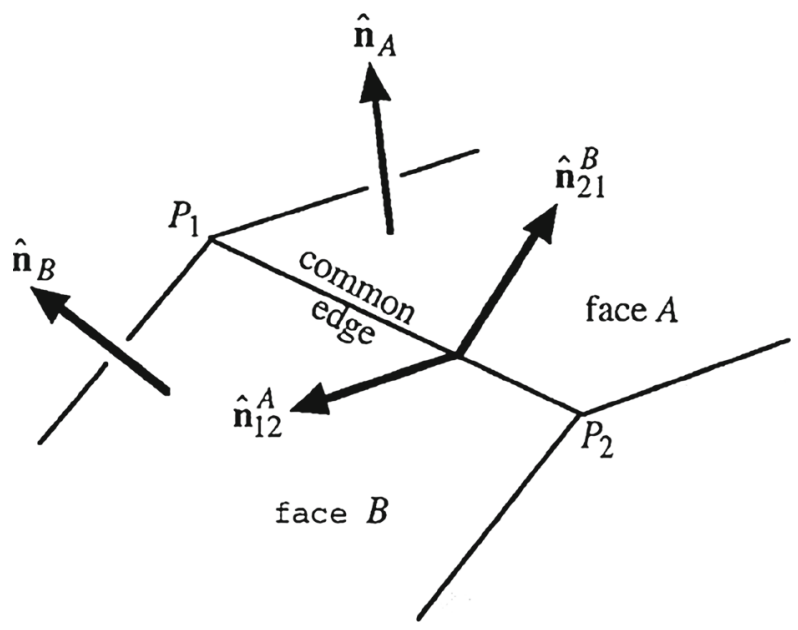

$$
\begin{aligned}
& \left(\frac{\partial U}{\partial \mathbf{r}}\right)^{T}=\mathbf{a}(\mathbf{r})=G \rho\left[-\sum_{e=1}^{N_{e}}\left[E_{e}\right] \mathbf{r}_{i_{e, 1}} L_{\mathrm{e}}+\sum_{f=1}^{N_{f}}\left[F_{f}\right] \mathbf{r}_{i_{f, 1}^{F}} \Omega_{\mathrm{f}}\right], \\
& {\left[\frac{\partial^{2} U}{\partial \mathbf{r}^{2}}\right]=\left[\frac{\partial \mathbf{a}}{\partial \mathbf{r}}\right]=G \rho\left[\sum_{e=1}^{N_{e}}\left[E_{e}\right] L_{\mathrm{e}}-\sum_{f=1}^{N_{f}}\left[F_{f}\right] \Omega_{\mathrm{f}}\right] .}
\end{aligned}
$$

The edge potential $L_{\mathrm{e}}$, performance factor $\Omega_{\mathrm{f}}$ and the other terms constituting these expressions are detailed below.

$$
\begin{aligned}
& \mathbf{r}_{i}=\mathbf{C}_{i}-\mathbf{r}, \\
& r_{i}=\left\|\mathbf{r}_{i}\right\|, \\
& L_{\mathrm{e}}=\ln \left(\frac{r_{i_{e, 1}}+r_{i_{e, 2}}+l_{e}}{r_{i_{e, 1}}+r_{i_{e, 2}}-l_{e}}\right), \\
& l_{e}=\left\|\mathbf{C}_{i_{e, 2}^{E}}-\mathbf{C}_{i_{e, 1}^{E} \|}\right\|, \\
& \Omega_{f}=2 \cdot \arctan 2\left(\mathbf{r}_{i_{f, 1}^{F}}^{T}\left(\mathbf{r}_{i_{f, 2}^{F}} \times \mathbf{r}_{i_{f, 3}^{F}}\right),\right. \\
&\left.r_{i_{f, 1}^{F}} r_{i_{f, 2}^{F}} r_{i_{f, 3}^{F}}+r_{i_{f, 1}^{F}} \mathbf{r}_{i_{f, 2}^{F}}^{T} \mathbf{r}_{i_{f, 3}^{F}}+r_{i_{f, 2}^{F}} \mathbf{r}_{i_{f, 3}^{F}}^{T} \mathbf{r}_{i_{f, 1}^{F}}+r_{i_{f, 3}^{F},} \mathbf{r}_{i_{f, 1}^{F}}^{T} \mathbf{r}_{i_{f, 2}^{F}}\right), \\
& {\left[F_{f}\right]=\hat{n}_{f} \hat{n}_{f}^{T} . }
\end{aligned}
$$

The edge dyad $\left[E_{e}\right]$ is defined as

$$
\left[E_{e}\right]=\hat{n}_{A}\left(\hat{n}_{12}^{A}\right)^{T}+\hat{n}_{B}\left(\hat{n}_{21}^{B}\right)^{T},
$$

following the notations of Fig. 1.

The PGM expressions remain valid within the Brillouin sphere of the object, unlike exterior spherical harmonics expansions that diverge once evaluated within it (Werner 1997). The PGM in its provided form is valid everywhere except on the edges of the considered shape, although modified PGM expressions dealing with these singularities do exist (Tsoulis and Petrovic 2001). 


\section{First variation in the PGM expressions}

The expressions of the first variations $\delta U(\mathbf{r})$ and $\delta \mathbf{a}(\mathbf{r})$ provide valuable insight into the evolution of these gravity terms under a change in the shape, in addition to providing the pathway towards linearized uncertainty quantification in these quantities.

\subsection{First variation in the potential due to a change in the shape}

Rewrite the potential as

$$
U(\mathbf{r})=\frac{G \rho}{2}\left[\sum_{e=1}^{N_{e}} U_{e}^{E}+\sum_{f=1}^{N_{f}} U_{f}^{F}\right],
$$

with

$$
U_{e}^{E}=\mathbf{r}_{i_{e, 1}^{E}}^{T}\left[E_{e}\right] \mathbf{r}_{i_{e, 1}} L_{\mathrm{e}} \quad U_{f}^{F}=-\mathbf{r}_{i_{f, 1}^{F}}^{T}\left[F_{f}\right] \mathbf{r}_{i_{f, 1}^{F}} \Omega_{\mathrm{f}} .
$$

Taking the first variation yields

$$
\delta U(\mathbf{r})=\frac{G \rho}{2}\left[\sum_{e=1}^{N_{e}} \delta U_{e}^{E}+\sum_{f=1}^{N_{f}} \delta U_{f}^{F}\right],
$$

with

$$
\delta U_{e}^{E}=\left(\begin{array}{c}
\mathbf{r}_{i_{e, 1}^{E}}^{T}\left[E_{e}\right] \mathbf{r}_{i_{e, 1}^{E}} \\
2 L_{e}\left[E_{e}\right] \mathbf{r}_{i_{e, 1}} \\
L_{e}\left[R_{i_{e, 1}^{E}}^{E}\right]^{T} \mathbf{r}_{i_{e, 1} E}^{T}
\end{array}\right)^{T}\left(\begin{array}{c}
\delta L_{e} \\
\delta \mathbf{r}_{i_{e, 1}^{E}} \\
\delta \mathbf{E}_{e}
\end{array}\right),
$$

where $\mathbf{E}_{e}$ and $\left[R_{i_{e, 1}^{E}}^{E}\right]$ are defined such that the equality $\left[E_{e}\right] \mathbf{r}_{i_{e, 1}^{E}}=\left[R_{i_{e, 1}^{E}}^{E}\right] \mathbf{E}_{e}$ holds. $\left[R_{i_{e, 1}^{E}}^{E}\right]$ is a matrix of size $3 \times 6$ constructed from the components of $\mathbf{r}_{i_{e, 1}}$, and $\mathbf{E}_{e} \in \mathbb{R}^{6}$ holds the unique components of $\left[E_{e}\right]$. Similarly,

$$
\delta U_{f}^{F}=-\left(\begin{array}{c}
\mathbf{r}_{i_{f, 1}^{F}\left[F_{f}\right] \mathbf{r}_{i_{f, 1}^{F}}} \\
2 \Omega_{f}\left[F_{f}\right] \mathbf{r}_{i_{f, 1}^{F}} \\
\Omega_{f}\left[R_{i_{f, 1}^{F}}^{F}\right]^{T} \mathbf{r}_{i_{f, 1}^{F}}
\end{array}\right)^{T}\left(\begin{array}{c}
\delta \Omega_{f} \\
\delta \mathbf{r}_{i_{f, 1}^{F}} \\
\delta \mathbf{F}_{f}
\end{array}\right),
$$

where again $\left[F_{f}\right] \mathbf{r}_{i_{f, 1}^{F}}=\left[R_{i_{f, 1}^{F}}^{F}\right] \mathbf{F}_{f} \cdot\left[R_{i_{f, 1}^{F}}^{F}\right]$ is a matrix of size $3 \times 6$ constructed from the components of $\mathbf{r}_{i_{f, 1}}$, and $\mathbf{F}_{f} \in \mathbb{R}^{6}$ holds the unique components of $\left[F_{f}\right]$. In so many words,

$$
\delta U_{e}^{E}=\left(\frac{\partial U_{e}^{E}}{\partial \mathbf{X}_{e}^{E}}\right) \delta \mathbf{X}_{e}^{E} \quad \delta U_{f}^{F}=\left(\frac{\partial U_{f}^{F}}{\partial \mathbf{X}_{f}^{F}}\right) \delta \mathbf{X}_{f}^{F},
$$

with $\mathbf{X}_{e}^{E} \in \mathbb{R}^{10}$ and $\mathbf{X}_{f}^{F} \in \mathbb{R}^{10}$ defined as

$$
\mathbf{X}_{e}^{E}=\left(\begin{array}{c}
L_{e} \\
\mathbf{r}_{i_{e, 1}^{E}} \\
\mathbf{E}_{e}
\end{array}\right) \quad \mathbf{X}_{f}^{F}=\left(\begin{array}{c}
\Omega_{f} \\
\mathbf{r}_{i_{f, 1}^{F}} \\
\mathbf{F}_{f}
\end{array}\right) .
$$


The following sections delve into the derivation of the first variations in the different partitions of $\mathbf{X}_{e}^{E}$ and $\mathbf{X}_{f}^{F}$ so as to relate them to the first variations in the underlying first variation in the stacked-up vertex coordinates, noted $\delta \mathbf{C}$.

\subsubsection{First variation in $\mathrm{X}_{f}^{F}$}

The first variation in $\mathbf{X}_{f}^{F}$ can be written as

$$
\delta \mathbf{X}_{f}^{F}=\left(\begin{array}{c}
\delta \Omega_{f} \\
\delta \mathbf{r}_{i_{f, 1}^{F}} \\
\delta \mathbf{F}_{f}
\end{array}\right)=\left[\frac{\partial \mathbf{X}_{f}^{F}}{\partial \mathbf{T}_{f}}\right] \delta \mathbf{T}_{f}=\left[\begin{array}{c}
\left(\partial \Omega_{f} / \partial \mathbf{T}_{f}\right) \\
{\left[\partial \mathbf{r}_{i_{f, 1}^{F}} / \partial \mathbf{T}_{f}\right]} \\
{\left[\partial \mathbf{F}_{f} / \partial \mathbf{T}_{f}\right]}
\end{array}\right] \delta \mathbf{T}_{f},
$$

where the current triangular facet is formed by three vertices whose coordinates are stacked in a vector $\mathbf{T}_{f} \in \mathbf{R}^{9}$

$$
\mathbf{T}_{f}=\left(\begin{array}{l}
\mathbf{C}_{i_{f, 1}^{F}} \\
\mathbf{C}_{i_{f, 2}^{F}} \\
\mathbf{C}_{i_{f, 3}^{F}}
\end{array}\right)
$$

The different partials are now defined.

Derivation of $\left(\partial \Omega_{f} / \partial \mathbf{T}_{f}\right)$ :

One must be wary of the use of the arctan 2 function when taking its derivative. Indeed, treating arctan2 like arctan and differentiating (8) will yield spurious results should the denominator and numerator be of different signs. Instead, define $\hat{r}_{i_{f, j}^{F}}=\mathbf{r}_{i_{f, j}^{F}} /\left\|\mathbf{r}_{i_{f, j}^{F}}\right\|(j \in$ $\{1,2,3\})$ and

$$
\begin{aligned}
\mathbf{Z}_{f} & =\left(\begin{array}{cc}
1+\hat{r}_{i_{f, 2}^{F}}^{T} & \hat{r}_{i_{f, 3}^{F}}+\hat{r}_{i_{f, 3}^{F}}^{T} \hat{r}_{i_{f, 1}^{F}}+\hat{r}_{i_{f, 1}^{F}}^{T} \hat{r}_{i_{f, 2}^{F}} \\
& \hat{r}_{i_{f, 1}^{T}}^{T}\left(\hat{r}_{i_{f, 2}^{F}} \times \hat{r}_{i_{f, 3}^{F}}\right)
\end{array}\right), \\
\hat{x} & =\left(\begin{array}{l}
1 \\
0
\end{array}\right) \quad \hat{y}=\left(\begin{array}{l}
0 \\
1
\end{array}\right),
\end{aligned}
$$

and use the following definition of $\arctan 2$ that makes uses of the half-angle trigonometric formulae:

$$
\arctan 2(\mathbf{Z})=2 \arctan \left(\frac{\hat{y}^{T} \mathbf{Z}}{\|\mathbf{Z}\|+\hat{x}^{T} \mathbf{Z}}\right) .
$$

Then, the performance factor simply becomes

$$
\Omega_{f}=4 \arctan \left(\frac{\hat{y}^{T} \mathbf{Z}_{f}}{\left\|\mathbf{Z}_{f}\right\|+\hat{x}^{T} \mathbf{Z}_{f}}\right),
$$

and its first variation is then given by

$$
\delta \Omega_{f}=4 \frac{\left[\left\|\mathbf{Z}_{f}\right\|+\hat{x}^{T} \mathbf{Z}_{f}\right] \hat{y}^{T}-\hat{y}^{T} \mathbf{Z}_{f}\left[\hat{x}^{T}+\frac{\mathbf{Z}_{f}^{T}}{\|\mathbf{Z}\|}\right]}{\left(\left\|\mathbf{Z}_{f}\right\|+\hat{x}^{T} \mathbf{Z}_{f}\right)^{2}+\left(\hat{y}^{T} \mathbf{Z}_{f}\right)^{2}} \delta \mathbf{Z}_{f},
$$


where

$$
\mathbf{r}_{f}=\left(\begin{array}{c}
\mathbf{r}_{i_{f, 1}^{F}} \\
\mathbf{r}_{i_{f, 2}^{F}} \\
\mathbf{r}_{i_{f, 3}^{F}}
\end{array}\right) \quad \hat{r}_{f}=\left(\begin{array}{c}
\hat{r}_{i_{f, 1}^{F}} \\
\hat{r}_{i_{f, 2}^{F}} \\
\hat{r}_{i_{f, 3}^{F}}
\end{array}\right)
$$

and

$$
\delta \mathbf{Z}_{f}=\left[\begin{array}{ll}
\hat{r}_{i_{f, 3}^{F}}+\hat{r}_{i_{f, 2}^{F}} & {\left[\hat{r}_{i_{f, 2}^{F}}\right]^{\times} \hat{r}_{i_{f, 3}^{F}}} \\
\hat{r}_{i_{f, 1}^{F}}+\hat{r}_{i_{f, 3}^{F}} & {\left[\hat{r}_{i_{f, 3}^{F}}\right]^{\times} \hat{r}_{i_{f, 1}^{F}}} \\
\hat{r}_{i_{f, 1}^{F}}+\hat{r}_{i_{f, 2}^{F}} & {\left[\hat{r}_{i_{f, 1}^{F}}\right]^{\times} \hat{r}_{i_{f, 2}^{F}}^{T}}
\end{array}\right]^{T}\left(\begin{array}{c}
\delta \hat{r}_{i_{f, 1}^{F}} \\
\delta \hat{r}_{i_{f, 2}^{F}} \\
\delta \hat{r}_{i_{f, 3}^{F}}^{F}
\end{array}\right) .
$$

The first variation in any of the $\hat{r}_{i_{f, j}^{F}}$ is given by

$$
\delta \hat{r}_{i_{f, j}^{F}}=\left(\frac{[I]_{3}}{\left\|\mathbf{r}_{i_{f, j}^{F}}\right\|}-\frac{\mathbf{r}_{i_{f, j}^{F}} \mathbf{r}_{i_{f, j}^{F}}^{T}}{\left\|\mathbf{r}_{i_{f, j}^{F}}\right\|^{3}}\right) \delta \mathbf{r}_{i_{f, j}^{F}}=\left[\frac{\partial \hat{r}_{i_{f, j}^{F}}}{\partial \mathbf{r}_{i_{f, j}^{F}}}\right] \delta \mathbf{r}_{i_{f, j}^{F}} .
$$

Finally, since $\mathbf{r}_{i_{f, j}^{F}}=\mathbf{C}_{i_{f, j}}-\mathbf{r}$, it is clear that

$$
\left(\begin{array}{l}
\delta \mathbf{r}_{i_{f, 1}^{F}} \\
\delta \mathbf{r}_{i_{f, 2}^{F}} \\
\delta \mathbf{r}_{i_{f, 3}^{F}}
\end{array}\right)=\left[\begin{array}{ccc}
{[I]_{3}} & {[0]_{3,3}} & {[0]_{3,3}} \\
{[0]_{3,3}} & {[I]_{3}} & {[0]_{3,3}} \\
{[0]_{3,3}} & {[0]_{3,3}} & {[I]_{3}}
\end{array}\right]\left(\begin{array}{l}
\delta \mathbf{C}_{i_{f, 1}^{F}} \\
\delta \mathbf{C}_{i_{f, 2}^{F}} \\
\delta \mathbf{C}_{i_{f, 3}^{F}}
\end{array}\right)=\left(\begin{array}{l}
\delta \mathbf{C}_{i_{f, 1}^{F}} \\
\delta \mathbf{C}_{i_{f, 2}^{F}} \\
\delta \mathbf{C}_{i_{f, 3}^{F}}
\end{array}\right)=\delta \mathbf{T}_{f} .
$$

Derivation of $\left[\partial \mathbf{r}_{i_{f, 1}^{F}} / \partial \mathbf{T}_{f}\right]$ : Since $\mathbf{r}_{i_{f, 1}^{F}}=\mathbf{C}_{i_{f, 1}^{F}}-\mathbf{r}$, the sought-for partial simply reads

$$
\left[\frac{\partial \mathbf{r}_{i_{f, 1}^{F}}}{\partial \mathbf{T}_{f}}\right]=\left[\begin{array}{lll}
{[I]_{3}} & {[0]_{3,3}} & {[0]_{3,3}}
\end{array}\right] .
$$

Derivation of $\left[\partial \mathbf{F}_{f} \partial \mathbf{T}_{f}\right]$ :

The facet dyad $\left[F_{f}\right]=\hat{n}_{f} \hat{n}_{f}^{T}$ is formed from the outer-product of the normalized surface normal with itself. It is obviously symmetric, so that it can be parametrized by

$$
\left[F_{f}\right]=\left[\begin{array}{ccc}
{\left[F_{f}\right](1,1)} & {\left[F_{f}\right](1,2)} & {\left[F_{f}\right](1,3)} \\
\cdot & {\left[F_{f}\right](2,2)} & {\left[F_{f}\right](2,3)} \\
. & \cdot & {\left[F_{f}\right](3,3)}
\end{array}\right] .
$$

It must be noted that for $(q, r) \in\{1,2,3\} \times\{1,2,3\}$

$$
\left[F_{f}\right](q, r)=\hat{e}_{q}^{T}\left[F_{f}\right] \hat{e}_{r} .
$$

So the vector-form parametrization of $\left[F_{f}\right]$ can be written as

$$
\mathbf{F}_{f}=\left(\begin{array}{l}
{\left[F_{f}\right](1,1)} \\
{\left[F_{f}\right](2,2)} \\
{\left[F_{f}\right](3,3)} \\
{\left[F_{f}\right](1,2)} \\
{\left[F_{f}\right](1,3)} \\
{\left[F_{f}\right](2,3)}
\end{array}\right)=\left(\begin{array}{l}
\hat{n}_{f}^{T} \hat{e}_{1} \hat{e}_{1}^{T} \hat{n}_{f} \\
\hat{n}_{f}^{T} \hat{e}_{2} \hat{e}_{2}^{T} \hat{n}_{f} \\
\hat{n}_{f}^{T} \hat{e}_{3} \hat{e}_{3}^{T} \hat{n}_{f} \\
\hat{n}_{f}^{T} \hat{e}_{1} \hat{e}_{2}^{T} \hat{n}_{f} \\
\hat{n}_{f}^{T} \hat{e}_{1} \hat{e}_{3}^{T} \hat{n}_{f} \\
\hat{n}_{f}^{T} \hat{e}_{2} \hat{e}_{3}^{T} \hat{n}_{f}
\end{array}\right) .
$$


Therefore,

$$
\delta \mathbf{F}_{f}=\left(\begin{array}{c}
2 \hat{n}_{f}^{T} \hat{e}_{1} \hat{e}_{1}^{T} \\
2 \hat{n}_{f}^{T} \hat{e}_{2} \hat{e}_{2}^{T} \\
2 \hat{n}_{f}^{T} \hat{e}_{3} \hat{e}_{3}^{T} \\
\hat{n}_{f}^{T}\left(\hat{e}_{1} \hat{e}_{2}^{T}+\hat{e}_{2} \hat{e}_{1}^{T}\right) \\
\hat{n}_{f}^{T}\left(\hat{e}_{1} \hat{e}_{3}^{T}+\hat{e}_{3} \hat{e}_{1}^{T}\right) \\
\hat{n}_{f}^{T}\left(\hat{e}_{2} \hat{e}_{3}^{T}+\hat{e}_{3} \hat{e}_{2}^{T}\right)
\end{array}\right) \delta \hat{n}_{f}=\left[\frac{\partial \mathbf{F}_{f}}{\partial \hat{n}_{f}}\right] \delta \hat{n}_{f} .
$$

Introducing the non-normalized surface normal $\hat{n}_{f}=\frac{\mathbf{n}_{f}}{\left\|\mathbf{n}_{f}\right\|}$ with

$$
\mathbf{n}_{f}=\left(\mathbf{C}_{i_{f, 2}^{F}}-\mathbf{C}_{i_{f, 1}^{F}}\right) \times\left(\mathbf{C}_{i_{f, 3}^{F}}-\mathbf{C}_{i_{f, 1}^{F}}\right),
$$

and since the partial derivative of an arbitrary unit vector $\hat{u}$ with respect to its non-normalized counterpart $\mathbf{u}$ is given by

$$
\delta \hat{u}=\left(\frac{[I]_{3}}{\|\mathbf{u}\|}-\frac{\mathbf{u} \mathbf{u}^{T}}{\|\mathbf{u}\|^{3}}\right) \delta \mathbf{u}=\left[\frac{\partial \hat{u}}{\partial \mathbf{u}}\right] \delta \mathbf{u},
$$

where

$$
\begin{aligned}
\delta \mathbf{n}_{f} & =\left[\begin{array}{lll}
{\left[\mathbf{C}_{i_{f, 3}^{F}}-\mathbf{C}_{i_{f, 2}^{F}}\right]^{\times}} & {\left[\mathbf{C}_{i_{f, 1}^{F}}-\mathbf{C}_{i_{f, 3}^{F}}\right]^{\times}} & {\left[\mathbf{C}_{i_{f, 2}^{F}}-\mathbf{C}_{i_{f, 1}^{F}}\right]^{\times}}
\end{array}\right]\left(\begin{array}{l}
\delta \mathbf{C}_{i_{f, 1}^{F}} \\
\delta \mathbf{C}_{i_{f, 2}^{F}} \\
\delta \mathbf{C}_{i_{f, 3}^{F}}^{F}
\end{array}\right) \\
& =\left[\frac{\partial \mathbf{n}_{f}}{\partial \mathbf{T}_{f}}\right] \delta \mathbf{T}_{f},
\end{aligned}
$$

the sought-for partial is given by

$$
\left[\frac{\partial \mathbf{F}_{f}}{\partial \mathbf{T}_{f}}\right]=\left[\frac{\partial \mathbf{F}_{f}}{\partial \hat{n}_{f}}\right]\left[\frac{\partial \hat{n}_{f}}{\partial \mathbf{n}_{f}}\right]\left[\frac{\partial \mathbf{n}_{f}}{\partial \mathbf{T}_{f}}\right] .
$$

\subsubsection{First variation in $\mathrm{X}_{e}^{E}$}

The coordinates of the two vertices forming the $e$-th edge are stacked in a vector $\mathbf{A}_{e} \in \mathbb{R}^{6}$ defined as

$$
\mathbf{A}_{e}=\left(\begin{array}{l}
\mathbf{C}_{i_{e, 1}^{E}} \\
\mathbf{C}_{i_{e, 2}}
\end{array}\right) .
$$

$\mathbf{T}_{i_{e, 1}^{E F}}, \mathbf{T}_{i_{e, 2}^{E F}}$ hold the coordinates of the vertices of the two facets adjacent to edge $e$. Defining $\mathbf{B}_{e} \in \mathbb{R}^{24}$ as

$$
\mathbf{B}_{e}=\left(\begin{array}{c}
\mathbf{A}_{e} \\
\mathbf{T}_{i_{e, 1}^{E F}} \\
\mathbf{T}_{i_{e, 2}^{E F}}
\end{array}\right)
$$

the first variation in $\mathbf{X}_{e}^{E}$ can be written as

$$
\delta \mathbf{X}_{e}^{E}=\left[\partial \mathbf{X}_{e}^{E} / \partial \mathbf{B}_{e}\right] \delta \mathbf{B}_{e}
$$


where the $10 \times 24$ matrix $\left[\partial \mathbf{X}_{e}^{E} / \partial \mathbf{B}_{e}\right]$ is defined as

$$
\left[\partial \mathbf{X}_{e}^{E} / \partial \mathbf{B}_{e}\right]=\left[\begin{array}{ccc}
\left(\partial L_{e} / \partial \mathbf{A}_{e}\right) & \mathbf{0}_{9}^{T} & \mathbf{0}_{9}^{T} \\
{\left[\partial \mathbf{r}_{i_{e, 1}} / \partial \mathbf{A}_{e}\right]} & 0_{39} & 0_{39} \\
& {\left[\partial \mathbf{E}_{e} / \partial \mathbf{B}_{e}\right]}
\end{array}\right]
$$

The different partials are now defined: Derivation of $\left(\partial L_{e} / \partial \mathbf{A}_{e}\right)$ :

From

$$
\begin{aligned}
L_{e} & =\ln \left(\frac{r_{i_{e, 1}}+r_{i_{e, 2}}+l_{e}}{r_{i_{e, 1}}+r_{i_{e, 2}}-l_{e}}\right) \\
\delta L_{e} & =\frac{\delta r_{i_{e, 1}}+\delta r_{i_{e, 2}}+\delta l_{e}}{r_{i_{e, 1}}+r_{i_{e, 2}}+l_{e}}-\frac{\delta r_{i_{e, 1}}+\delta r_{i_{e, 2}}-\delta l_{e}}{r_{i_{e, 1}}+r_{i_{e, 2}}-l_{e}},
\end{aligned}
$$

writing

$$
\beta_{e}^{+}=r_{i_{e, 1}}+r_{i_{e, 2}}+l_{e} \beta_{e}^{-}=r_{i_{e, 1}}+r_{i_{e, 2}}-l_{e},
$$

this first variation becomes

$$
\begin{aligned}
\delta L_{e} & =\frac{\delta r_{i_{e, 1}}+\delta r_{i_{e, 2}}+\delta l_{e}}{\beta_{e}^{+}}-\frac{\delta r_{i_{e, 1}}+\delta r_{i_{e, 2}}-\delta l_{e}}{\beta_{e}^{-}} \\
& =\left(\begin{array}{l}
1 / \beta_{e}^{+}+1 / \beta_{e}^{-} \\
1 / \beta_{e}^{+}-1 / \beta_{e}^{-} \\
1 / \beta_{e}^{+}-1 / \beta_{e}^{-}
\end{array}\right)^{T}\left(\begin{array}{c}
\delta l_{e} \\
\delta r_{i_{e, 1}} \\
\delta r_{i_{e, 2}}
\end{array}\right) .
\end{aligned}
$$

Since

$$
\delta l_{e}=\frac{\left(\mathbf{C}_{i_{e, 1}}-\mathbf{C}_{i_{e, 2}}\right)^{T}}{l_{e}}\left[[I]_{3}-[I]_{3}\right] \delta \mathbf{A}_{e}=\left(\frac{\partial l_{e}}{\partial \mathbf{A}_{e}}\right) \delta \mathbf{A}_{e}
$$

and

$$
\frac{\partial \mathbf{r}_{i_{e, 1}^{E}}}{\partial \mathbf{A}_{e}}=\left[\begin{array}{ll}
{[I]_{3}} & {[0]_{3,3}}
\end{array}\right] \quad \frac{\partial \mathbf{r}_{i_{e, 2}}}{\partial \mathbf{A}_{e}}=\left[\begin{array}{ll}
{[0]_{3,3}} & {[I]_{3}}
\end{array}\right],
$$

the first variation in $L_{e}$ finally becomes

$$
\begin{aligned}
\delta L_{e}= & \left(\frac{\partial L_{e}}{\partial \mathbf{A}_{e}}\right) \delta \mathbf{A}_{e} \\
= & \left(\begin{array}{l}
1 / \beta_{e}^{+}+1 / \beta_{e}^{-} \\
1 / \beta_{e}^{+}-1 / \beta_{e}^{-} \\
1 / \beta_{e}^{+}-1 / \beta_{e}^{-}
\end{array}\right)^{T}\left[\begin{array}{c}
\left(\partial l_{e} / \partial \mathbf{A}_{e}\right) \\
\left(\partial r_{i_{e, 1}} / \partial \mathbf{r}_{i_{e, 1}}\right)\left[\partial \mathbf{r}_{i_{e, 1}^{E}} / \partial \mathbf{A}_{e}\right] \\
\left(\partial r_{i_{e, 2}} / \partial \mathbf{r}_{i_{e, 2}}\right)\left[\partial \mathbf{r}_{i_{e, 2}^{E}} / \partial \mathbf{A}_{e}\right]
\end{array}\right] \delta \mathbf{A}_{e} .
\end{aligned}
$$

Derivation of $\left[\partial \mathbf{r}_{i_{e, 1}} / \partial \mathbf{A}_{e}\right]$ : Since $\mathbf{r}_{i_{e, 1}^{E}}=\mathbf{C}_{i_{e, 1}^{E}}-\mathbf{r}$, the sought-for partial simply reads

$$
\left[\frac{\partial \mathbf{r}_{i_{e, 1}}}{\partial \mathbf{A}_{e}}\right]=\left[\begin{array}{ll}
{[I]_{3}} & {[0]_{3,3}}
\end{array}\right] \text {. }
$$

Derivation of $\left[\partial \mathbf{E}_{e} / \partial \mathbf{B}_{e}\right]$ : following Werner and Scheeres' notation, the edge dyad $\left[E_{e}\right]$ is formed from

$$
\left[E_{e}\right]=\hat{n}_{A} \hat{n}_{12}^{A}+\hat{n}_{B} \hat{n}_{21}^{B} .
$$


The two vertices forming the $e$-th edge of length $l_{e}$ being $\mathbf{C}_{i_{e, 1}}$ and $\mathbf{C}_{i_{e, 2}}$, the closed-form expression of the $e$-th edge dyad becomes

$$
\left[E_{e}\right]=\frac{1}{l_{e}}\left(\hat{n}_{i_{e, 2}^{E F}} \hat{n}_{i_{e, 2}^{E F F}}^{T}-\hat{n}_{i_{e, 1}^{E F}} \hat{n}_{i_{e, 1}^{E F}}^{T}\right)\left[\mathbf{C}_{i_{e, 2}^{E}}-\mathbf{C}_{i_{e, 1}^{E}}\right]^{\times} .
$$

Here, the index $f_{e, j}^{E F}$ with $j \in\{1,2\}$ refers to the index of the $j$-th facet associated with the $e$-th edge (an edge being effectively defined as the boundary between two facets). It is remarkable that $\left[E_{e}\right]$ is a symmetric tensor (Werner and Scheeres 1997). It can thus be parametrized by

$$
\left[E_{e}\right]=\left[\begin{array}{ccc}
{\left[E_{e}\right](1,1)} & {\left[E_{e}\right](1,2)} & {\left[E_{e}\right](1,3)} \\
\cdot & {\left[E_{e}\right](2,2)} & {\left[E_{e}\right](2,3)} \\
\cdot & \cdot & {\left[E_{e}\right](3,3)}
\end{array}\right] .
$$

Again, we have

$$
\left[E_{e}\right](q, r)=\hat{e}_{q}^{T}\left[E_{e}\right] \hat{e}_{r} .
$$

So the vector-form parametrization of $\left[E_{e}\right]$ can be written as

$$
\mathbf{E}_{e}=\left(\begin{array}{l}
{\left[E_{e}\right](1,1)} \\
{\left[E_{e}\right](2,2)} \\
{\left[E_{e}\right](3,3)} \\
{\left[E_{e}\right](1,2)} \\
{\left[E_{e}\right](1,3)} \\
{\left[E_{e}\right](2,3)}
\end{array}\right)=\left(\begin{array}{l}
\hat{e}_{1}^{T}\left[E_{e}\right] \hat{e}_{1} \\
\hat{e}_{2}^{T}\left[E_{e}\right] \hat{e}_{2} \\
\hat{e}_{3}^{T}\left[E_{e}\right] \hat{e}_{3} \\
\hat{e}_{1}^{T}\left[E_{e}\right] \hat{e}_{2} \\
\hat{e}_{1}^{T}\left[E_{e}\right] \hat{e}_{3} \\
\hat{e}_{2}^{T}\left[E_{e}\right] \hat{e}_{3}
\end{array}\right)
$$

Given an arbitrary component of the edge dyad $\left[E_{e}\right](q, r)=\hat{e}_{q}^{T}\left[E_{e}\right] \hat{e}_{r}$ can be written

$$
\left[E_{e}\right](q, r)=\frac{1}{l_{e}} \hat{e}_{q}^{T}\left(\hat{n}_{i_{e, 2}^{E F}} \hat{n}_{i_{e, 2}^{T E}}^{T}-\hat{n}_{i_{e, 1}^{E F}} \hat{n}_{i_{e, 1}^{E F}}^{T}\right)\left[\mathbf{C}_{i_{e, 2}^{E}}-\mathbf{C}_{i_{e, 1}}\right]^{\times} \hat{e}_{r}
$$

The first variation in $\left[E_{e}\right](q, r)$ can be expressed as

$$
\begin{aligned}
& \delta\left[E_{e}\right](q, r)=\frac{1}{l_{e}}\left[\begin{array}{c}
-\left[E_{e}\right](q, r) \\
M^{T}\left[\hat{e}_{r}\right]^{\times}\left[\left(\hat{e}_{q}^{T} \hat{n}_{i_{e, 2}^{E F}}\right) \hat{n}_{i_{e, 2}^{E F}}-\left(\hat{e}_{q}^{T} \hat{n}_{i_{e, 1} E F}\right) \hat{n}_{i_{e, 1} E F}\right] \\
-\left[\hat{e}_{q} \hat{n}_{i_{e, 1}^{T E F}}^{T}+\left(\hat{e}_{q}^{T} \hat{n}_{i_{e, 1} E F}\right)[I]_{3}\right] \mathbf{V}_{r} \\
{\left[\hat{e}_{q} \hat{n}_{i_{e, 2}^{E F}}^{T}+\left(\hat{e}_{q}^{T} \hat{n}_{i_{e, 2}^{E F}}\right)[I]_{3}\right] \mathbf{V}_{r}}
\end{array}\right]^{T} \\
& \cdot\left[\begin{array}{ccc}
\left(\partial l_{e} / \partial \mathbf{A}_{e}\right) & \mathbf{0}_{9}^{T} & \mathbf{0}_{9}^{T} \\
{[I]_{6}} & {[0]_{6,9}} & {[0]_{6,9}} \\
{[0]_{3,6}} & {\left[\partial \hat{n}_{f_{e, 1}^{E F}} / \partial \mathbf{T}_{f_{e, 1}^{E F}}\right]} & {[0]_{3,9}} \\
{[0]_{3,6}} & {[0]_{3,9}} & {\left[\partial \hat{n}_{f_{e, 2}^{E F}} / \partial \mathbf{T}_{f_{e, 2}^{E F}}\right]}
\end{array}\right] \delta \mathbf{B}_{e},
\end{aligned}
$$


with $M=\left[-[I]_{3} \quad[I]_{3}\right]$ and $\mathbf{V}_{r}=\left[\mathbf{C}_{i_{e, 2}^{E}}-\mathbf{C}_{i_{e, 1}^{E E}}\right]^{\times} \hat{e}_{r}$. Since $\hat{n}_{i_{e, 1}^{E F}}=\frac{\mathbf{n}_{i_{e, j}^{E F}}}{\left\|\mathbf{n}_{i, e F}^{E F}\right\|}$,

$$
\left[\partial \hat{n}_{f_{e, j}^{E F}} / \partial \mathbf{T}_{f_{e, j}^{E F}}\right]=\left[\partial \hat{n}_{f_{e, j}^{E F}} / \mathbf{n}_{f_{e, j}^{E F}}\right]\left[\partial \mathbf{n}_{f_{e, j}^{E F}} / \partial \mathbf{T}_{f_{e, j}^{E F}}\right],
$$

with

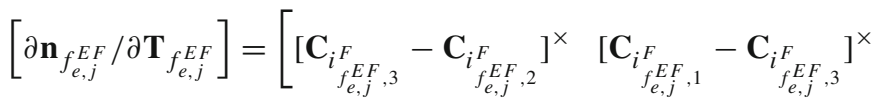

$$
\begin{aligned}
& \left.\left[\mathbf{C}_{i_{f_{e, j}^{E F}, 2}^{F F}}-\mathbf{C}_{f_{f_{e, j}^{E F}, 1}^{E F}}\right]^{\times}\right] \text {. }
\end{aligned}
$$

So the first variation in the parametrization of $\left[E_{e}\right]$ is given by

$$
\begin{aligned}
\delta \mathbf{E}_{e}= & {\left[\frac{\partial \mathbf{E}_{e}}{\partial \mathbf{B}_{e}}\right] \delta \mathbf{B}_{e} } \\
= & \left(\begin{array}{c}
\left(\partial E(1,1) / \partial \mathbf{B}_{e}\right) \\
\left(\partial E(2,2) / \partial \mathbf{B}_{e}\right) \\
\left(\partial E(3,3) / \partial \mathbf{B}_{e}\right) \\
\left(\partial E(1,2) / \partial \mathbf{B}_{e}\right) \\
\left(\partial E(1,3) / \partial \mathbf{B}_{e}\right) \\
\left(\partial E(2,3) / \partial \mathbf{B}_{e}\right)
\end{array}\right) \delta \mathbf{B}_{e} .
\end{aligned}
$$

\subsection{First variation in the acceleration due to a change in the shape}

From

$$
\mathbf{a}(\mathbf{r})=G \rho\left[\sum_{e=1}^{N_{e}} \mathbf{a}_{e}^{E}+\sum_{f=1}^{N_{f}} \mathbf{a}_{f}^{F}\right]
$$

with

$$
\mathbf{a}_{e}^{E}=-\left[E_{e}\right] \mathbf{r}_{i_{e, 1}^{E}} L_{e} \quad \mathbf{a}_{f}^{F}=\left[F_{f}\right] \mathbf{r}_{i_{f, 1}^{F}} \Omega_{f},
$$

the first variation in the acceleration caused by a change in the shape is given by

$$
\begin{aligned}
\delta \mathbf{a}(\mathbf{r}) & =G \rho\left[\sum_{e=1}^{N_{e}} \delta \mathbf{a}_{e}^{E}+\sum_{f=1}^{N_{f}} \delta \mathbf{a}_{f}^{F}\right] \\
& =G \rho\left[\sum_{e=1}^{N_{e}}\left[\frac{\partial \mathbf{a}_{e}^{E}}{\partial \mathbf{C}}\right]+\sum_{f=1}^{N_{f}}\left[\frac{\partial \mathbf{a}_{f}^{F}}{\partial \mathbf{C}}\right]\right] \delta \mathbf{C} \\
& =\left[\frac{\partial \mathbf{a}}{\partial \mathbf{C}}\right] \delta \mathbf{C},
\end{aligned}
$$


where

$$
\begin{aligned}
& \delta \mathbf{a}_{e}^{E}=\left[\begin{array}{lll}
{\left[E_{e}\right] \mathbf{r}_{i_{e, 1}^{E}}} & L_{e}\left[E_{e}\right] & L_{e} R_{i_{e, 1}^{E}}^{E}
\end{array}\right]\left(\begin{array}{c}
\delta L_{e} \\
\delta \mathbf{r}_{i_{e, 1}^{E}} \\
\delta \mathbf{E}_{e}
\end{array}\right)=\left[\begin{array}{c}
\frac{\partial \mathbf{a}_{e}^{E}}{\partial \mathbf{X}_{e}^{E}}
\end{array}\right] \delta \mathbf{X}_{e}^{E} \\
& =\left[\frac{\partial \mathbf{a}_{e}^{E}}{\partial \mathbf{X}_{e}^{E}}\right]\left[\frac{\partial \mathbf{X}_{e}^{E}}{\partial \mathbf{B}_{e}^{E}}\right]\left[\frac{\partial \mathbf{B}_{e}^{E}}{\partial \mathbf{C}}\right] \delta \mathbf{C},
\end{aligned}
$$

and

$$
\begin{aligned}
& \delta \mathbf{a}_{f}^{F}=\left[\begin{array}{lll}
{\left[F_{f}\right] \mathbf{r}_{i_{f, 1}^{F}}} & \Omega_{f}\left[F_{f}\right] & \Omega_{f} R_{i_{f, 1}^{F}}^{F}
\end{array}\right]\left(\begin{array}{c}
\delta \Omega_{f} \\
\delta \mathbf{r}_{i_{f, 1}^{F}} \\
\delta \mathbf{F}_{f}
\end{array}\right)=\left[\begin{array}{c}
\partial \mathbf{a}_{f}^{F} \\
\hline \partial \mathbf{X}_{f}^{F}
\end{array}\right] \delta \mathbf{X}_{f}^{F} \\
& =\left[\frac{\partial \mathbf{a}_{f}^{F}}{\partial \mathbf{X}_{f}^{F}}\right]\left[\frac{\partial \mathbf{X}_{f}^{F}}{\partial \mathbf{T}_{f}}\right]\left[\frac{\partial \mathbf{T}_{f}}{\partial \mathbf{C}}\right] \delta \mathbf{C} .
\end{aligned}
$$

\section{Second moment about the mean of the PGM quantities}

\subsection{Variance in potential of polyhedron gravity model}

From

$$
U(\mathbf{r})=\frac{G \rho}{2}\left[\sum_{e=1}^{N_{e}} U_{e}^{E}+\sum_{f=1}^{N_{f}} U_{f}^{F}\right]
$$

with

$$
U_{e}^{E}=\mathbf{r}_{i_{e, 1}^{E}}^{T}\left[E_{e}\right] \mathbf{r}_{i_{e, 1}^{E}} L_{e} \quad U_{f}^{F}=-\mathbf{r}_{i_{f, 1}^{F}}^{T}\left[F_{f}\right] \mathbf{r}_{i_{f, 1}^{F}} \Omega_{f},
$$

the first variation in the potential caused by a change in the shape is given by

$$
\delta U(\mathbf{r})=\frac{G \rho}{2}\left[\sum_{e=1}^{N_{e}}\left(\frac{\partial U_{e}^{E}}{\partial \mathbf{C}}\right)+\sum_{f=1}^{N_{f}}\left(\frac{\partial U_{f}^{F}}{\partial \mathbf{C}}\right)\right] \delta \mathbf{C}=\left(\frac{\partial U}{\partial \mathbf{C}}\right) \delta \mathbf{C},
$$

where

$$
\left(\frac{\partial U_{e}^{E}}{\partial \mathbf{C}}\right)=\left(\frac{\partial U_{e}^{E}}{\partial \mathbf{X}_{e}^{E}}\right)\left[\frac{\partial \mathbf{X}_{e}^{E}}{\partial \mathbf{B}_{e}^{E}}\right]\left[\frac{\partial \mathbf{B}_{e}^{E}}{\partial \mathbf{C}}\right]
$$

and

$$
\left(\frac{\partial U_{f}^{F}}{\partial \mathbf{C}}\right)=\left(\frac{\partial U_{f}^{F}}{\partial \mathbf{X}_{f}^{F}}\right)\left[\frac{\partial \mathbf{X}_{f}^{F}}{\partial \mathbf{T}_{f}}\right]\left[\frac{\partial \mathbf{T}_{f}}{\partial \mathbf{C}}\right] .
$$

The variance in the gravitational potential at a fixed point in space $\mathbf{r}$ is given by

$$
\sigma_{U U}^{2}(\mathbf{r})=\left(\frac{\partial U}{\partial \mathbf{C}}\right)\left[P_{\mathbf{C C}}\right]\left(\frac{\partial U}{\partial \mathbf{C}}\right)^{T},
$$

where $\left[P_{\mathbf{C C}}\right]$ is the $3 N_{C} \times 3 N_{C}$ covariance of the shape vertex coordinates. 


\subsection{Acceleration covariance}

The first variation in the acceleration caused by a change in the shape is given by

$$
\delta \mathbf{a}(\mathbf{r})=\frac{G \rho}{2}\left[\sum_{e=1}^{N_{e}}\left[\frac{\partial \mathbf{a}_{e}^{E}}{\partial \mathbf{C}}\right]+\sum_{f=1}^{N_{f}}\left[\frac{\partial \mathbf{a}_{f}^{F}}{\partial \mathbf{C}}\right]\right] \delta \mathbf{C}=\left[\frac{\partial \mathbf{a}}{\partial \mathbf{C}}\right] \delta \mathbf{C},
$$

where

$$
\left[\frac{\partial \mathbf{a}_{e}^{E}}{\partial \mathbf{C}}\right]=\left[\frac{\partial \mathbf{a}_{e}^{E}}{\partial \mathbf{X}_{e}^{E}}\right]\left[\frac{\partial \mathbf{X}_{e}^{E}}{\partial \mathbf{B}_{e}^{E}}\right]\left[\frac{\partial \mathbf{B}_{e}^{E}}{\partial \mathbf{C}}\right]
$$

and

$$
\left[\frac{\partial \mathbf{a}_{f}^{F}}{\partial \mathbf{C}}\right]=\left[\frac{\partial \mathbf{a}_{f}^{F}}{\partial \mathbf{X}_{f}^{F}}\right]\left[\frac{\partial \mathbf{X}_{f}^{F}}{\partial \mathbf{T}_{f}}\right]\left[\frac{\partial \mathbf{T}_{f}}{\partial \mathbf{C}}\right] .
$$

The covariance in the acceleration at a given point in space is given by

$$
\left[P_{\mathbf{a a}}(\mathbf{r})\right]=\left[\frac{\partial \mathbf{a}}{\partial \mathbf{C}}\right]\left[P_{\mathbf{C C}}\right]\left[\frac{\partial \mathbf{a}}{\partial \mathbf{C}}\right]^{T}
$$

\section{Surface slopes}

The surface slope at the center of facet $f$ is defined as

$$
s_{f}=\arccos \left(-\hat{b}_{f}^{T} \hat{n}_{f}\right)=\arccos (-u),
$$

$s_{f}$ is equal to 0 if the body-fixed acceleration direction $\hat{b}_{f}=\mathbf{b}_{f} /\left\|\mathbf{b}_{f}\right\|$ evaluated at the center of the facet $\mathbf{P}_{f}$ and the facet normal direction $\hat{n}_{f}$ are in opposite, anti-parallel directions. The body-fixed acceleration at the center of the $f$-th facet is given by

$$
\mathbf{b}_{f}\left(\mathbf{P}_{f}, \mathbf{C}\right)=\boldsymbol{a}_{f}\left(\mathbf{P}_{f}, \mathbf{C}\right)-\boldsymbol{\omega} \times\left(\boldsymbol{\omega} \times\left(\mathbf{P}_{f}-\mathbf{G}\right)\right),
$$

where $\boldsymbol{a}_{f}$ is the inertial gravity acceleration at $\mathbf{P}_{f}, \mathbf{G}$ the barycenter of the small body, and $\boldsymbol{\omega}$ its angular velocity. The accelerations are functions of both the facet center coordinates $\mathbf{P}_{f}$ and the shape vertex coordinates $\mathbf{C}$ since these dictate the overall gravity field. Surface slopes are of utmost interest to small body science and engineering since they may be indicative of areas where material can settle on the small body surface (Scheeres et al. 2006a, b; Nolan et al. 2013). The next section offers a short summary of the quantities of interest when investigating the variation in the slopes caused by uncertainties in the shape vertex coordinates and the small body rotation period. The actual expression of the first variation in the shape is then derived. The final deliverable of this section is the expression of the first variation in the slope as a function of two independent variables, $\delta \mathbf{C}$ and $\delta \boldsymbol{\omega}$, the latter being the first variation in the small body angular velocity magnitude.

\subsection{Inertia quantities of interest}

The surface slope at the center of facet $s_{f}$ is obviously tied to the local geometry through the facet normal $\hat{n}_{f}$, but also to the position of the center-of-mass of the body since the latter 
is undergoing rotation about an axis that goes through this point. This remark essentially ties together three important inertia quantities: the volume of the small body, its center-ofmass and its inertia tensor. Expressions of these quantities are thus provided to the reader in this section before moving on. Computing the volume, center-of-mass and inertia tensor of a constant density polyhedron boils down to accumulating the contribution of each of the $N_{f}$ facets-instead, of each tetrahedron subtended by the facet-in an orderly fashion (Dobrovolskis 1996). The expressions in this section are specialized from the expressions in (Bercovici and Mcmahon 2019) to triangular, planar, first-order surface elements.

\subsubsection{Volume}

The total volume of the polyhedron is given by

$$
V=\sum_{f=1}^{N_{f}} V_{f},
$$

where $V_{f}=\frac{1}{6} \cdot \operatorname{det}\left(\left[\begin{array}{lll}\mathbf{C}_{i_{f, 1}^{F}} & \mathbf{C}_{i_{f, 2}^{F}} & \mathbf{C}_{i_{f, 3}^{F}}\end{array}\right]\right)$ designates the signed volume of the considered tetrahedron (Dobrovolskis 1996).

\subsubsection{Center of mass}

The coordinates of the constant-density polyhedron barycenter are given by

$$
\mathbf{G}=\frac{1}{V} \sum_{f=1}^{N_{f}} V_{f} \mathbf{G}_{f},
$$

where $\mathbf{G}_{f}$ stands for the barycenter of the tetrahedron subtended by the $f$-th facet (Dobrovolskis 1996)

$$
\mathbf{G}_{f}=\frac{1}{4}\left(\mathbf{C}_{i_{f, 1}^{F}}+\mathbf{C}_{i_{f, 2}^{F}}+\mathbf{C}_{i_{f, 3}^{F}}\right)=\frac{1}{4} A \mathbf{T}_{f},
$$

with $A=\left[[I]_{3}[I]_{3}[I]_{3}\right]$.

\subsubsection{Inertia tensor}

The unit-density inertia tensor of the whole shape about $(0,0,0)^{T}$ is given by Bercovici and Mcmahon (2019)

$$
[I] \mathbf{o}=\sum_{f=1}^{N_{f}}[I] \mathbf{o}, f,
$$

where the contribution to the inertia tensor of every tetrahedron can be written as

$$
[I]_{\mathbf{O}, f}=\left[\frac{[I]_{\mathbf{o}, f}}{V_{f}}\right] V_{f},
$$


and

$$
\begin{aligned}
{\left[\frac{[I]_{\mathbf{o}, f}}{V_{f}}\right] } & =-\frac{1}{20}\left(\left[\mathbf{C}_{f, 1}+\mathbf{C}_{f, 2}+\mathbf{C}_{f, 3}\right]^{\times 2}+\left[\mathbf{C}_{f, 1}\right]^{\times 2}+\left[\mathbf{C}_{f, 2}\right]^{\times 2}+\left[\mathbf{C}_{f, 3}\right]^{\times 2}\right) \\
& =-\frac{1}{20}\left(\left[[A] \mathbf{T}_{f}\right]^{\times 2}+\left[\left[A_{0}\right] \mathbf{T}_{f}\right]^{\times 2}+\left[\left[A_{1}\right] \mathbf{T}_{f}\right]^{\times 2}+\left[\left[A_{2}\right] \mathbf{T}_{f}\right]^{\times 2}\right),
\end{aligned}
$$

where $\times 2$ in the exponents denote squaring the corresponding cross-product matrices, and

$$
\left[A_{0}\right]=\left[[I]_{3}[0]_{3,3}[0]_{3,3}\right] \quad\left[A_{1}\right]=\left[[0]_{3,3}[I]_{3}[0]_{3,3}\right] \quad\left[A_{2}\right]=\left[[0]_{3,3}[0]_{3,3}[I]_{3}\right]
$$

The parametrization of the inertia tensor is denoted $\mathbf{I}$ :

$$
\mathbf{I}=\left(\begin{array}{l}
{[I]_{\mathbf{O}}(1,1)} \\
{[I]_{\mathbf{O}}(2,2)} \\
{[I]_{\mathbf{O}}(3,3)} \\
{[I]_{\mathbf{O}}(1,2)} \\
{[I]_{\mathbf{O}}(1,3)} \\
{[I]_{\mathbf{O}}(2,3)}
\end{array}\right)=\sum_{f=1}\left(\begin{array}{l}
{[I]_{\mathbf{O}, f}(1,1)} \\
{[I]_{\mathbf{O}, f}(2,2)} \\
{[I]_{\mathbf{O}, f}(3,3)} \\
{[I]_{\mathbf{O}, f}(1,2)} \\
{[I]_{\mathbf{O}, f}(1,3)} \\
{[I]_{\mathbf{O}, f}(2,3)}
\end{array}\right)
$$

with $[I]_{\mathbf{O}}(q, r)=\hat{e}_{q}^{T}[I]_{\mathbf{O}} \hat{e}_{r}$ for $(q, r) \in\{1,2,3\} \times\{1,2,3\}$

\subsection{Partial derivative of the inertia quantities of interest}

The expressions of the partial derivatives of the quantities of interest, specialized to triangular, planar, first-order surface elements are given hereunder.

\subsubsection{Volume}

The partial derivative in the total volume relative to the shape is given by

$$
\left(\frac{\partial V}{\partial \mathbf{C}}\right)=\left[\sum_{f=1}^{N_{f}}\left(\frac{\partial V_{f}}{\partial \mathbf{C}}\right)\right]
$$

where

$$
\begin{gathered}
\left(\frac{\partial V_{f}}{\partial \mathbf{C}}\right)=\frac{1}{6}\left(\left(\mathbf{C}_{i_{f, 2}^{F}} \times \mathbf{C}_{i_{f, 3}^{F}}\right)^{T}\left(\mathbf{C}_{i_{f, 3}^{F}} \times \mathbf{C}_{i_{f, 1}^{F}}\right)^{T}\right. \\
\left.\left(\mathbf{C}_{i_{f, 1}^{F}} \times \mathbf{C}_{i_{f, 2}^{F}}\right)^{T}\right)\left[\frac{\partial \mathbf{T}_{f}}{\partial \mathbf{C}}\right] .
\end{gathered}
$$

\subsubsection{Center of mass}

The partial derivative of the barycenter with respect to the vertex coordinates is readily given by

$$
\left[\frac{\partial \mathbf{G}}{\partial \mathbf{C}}\right]=\frac{1}{V} \sum_{f=1}^{N_{f}}\left[\left(\mathbf{G}_{f}-\mathbf{G}\right)\left(\frac{\partial V_{f}}{\partial \mathbf{T}_{f}}\right)+V_{f}\left[\frac{\partial \mathbf{G}_{f}}{\partial \mathbf{T}_{f}}\right]\right]\left[\frac{\partial \mathbf{T}_{f}}{\partial \mathbf{C}}\right] .
$$




\subsubsection{Inertia tensor parametrization}

The first variation in the inertia tensor parametrization is written as

$$
\delta \mathbf{I}=\left(\begin{array}{l}
\delta[I]_{\mathbf{O}}(1,1) \\
\delta[I]_{\mathbf{O}}(2,2) \\
\delta[I]_{\mathbf{O}}(3,3) \\
\delta[I]_{\mathbf{O}}(1,2) \\
\delta[I]_{\mathbf{O}}(1,3) \\
\delta[I]_{\mathbf{O}}(2,3)
\end{array}\right)=\sum_{f=1}^{N_{f}}\left(\begin{array}{l}
\delta[I]_{\mathbf{O}, f}(1,1) \\
\delta[I]_{\mathbf{O}, f}(2,2) \\
\delta[I]_{\mathbf{O}, f}(3,3) \\
\delta[I]_{\mathbf{O}, f}(1,2) \\
\delta[I]_{\mathbf{O}, f}(1,3) \\
\delta[I]_{\mathbf{O}, f}(2,3)
\end{array}\right),
$$

where

$$
\begin{aligned}
\delta[I]_{\mathbf{O}, f}(q, r)= & \delta\left(V_{f} \hat{e}_{q}^{T}\left[\frac{[I]_{\mathbf{o}, f}}{V_{f}}\right] \hat{e}_{r}\right) \\
= & {\left[\hat{e}_{q}^{T}\left[\frac{[I]_{\mathbf{o}, f}}{V_{f}}\right] \hat{e}_{r}\left[\frac{\partial V_{f}}{\partial \mathbf{T}_{f}}\right]+V_{f}\left[\frac{\partial\left(\hat{e}_{q}^{T}\left[\frac{[I]_{0}, f}{V_{f}}\right] \hat{e}_{r}\right)}{\partial \mathbf{T}_{f}}\right]\right] } \\
& \cdot\left[\frac{\partial \mathbf{T}_{f}}{\partial \mathbf{C}}\right] \delta \mathbf{C} .
\end{aligned}
$$

Definining the $3 \times 3$ matrix $K_{q r}=\left(\left[\hat{e}_{q}\right]^{\times}\left[\hat{e}_{r}\right]^{\times}+\left[\hat{e}_{r}\right]^{\times}\left[\hat{e}_{q}\right]^{\times}\right)$, we have

$$
\begin{aligned}
& {\left[\frac{\partial\left(\hat{e}_{q}^{T}\left[\frac{[I]_{\mathbf{O}, f}}{V_{f}}\right] \hat{e}_{r}\right)}{\partial \mathbf{T}_{f}}\right]=-\frac{1}{20} \mathbf{T}_{f}^{T}\left[A^{T} K_{q r} A\right.} \\
& \left.+A_{0}^{T} K_{q r} A_{0}+A_{1}^{T} K_{q r} A_{1}+A_{2}^{T} K_{q r} A_{2}\right] .
\end{aligned}
$$

\subsection{Partial derivative of the surface slope relative to the shape coordinates and attitude}

Take the first variation in Eq. (81),

$$
\delta s_{f}=\frac{\delta u}{\sqrt{1-u^{2}}},
$$

and

$$
\begin{aligned}
\delta u & =\hat{n}_{f}^{T} \delta \hat{b}_{f}+\hat{b}_{f}^{T} \delta \hat{n}_{f}=\left(\begin{array}{c}
\hat{n}_{f} \\
\hat{b}_{f}
\end{array}\right)^{T}\left(\begin{array}{l}
\delta \hat{b}_{f} \\
\delta \hat{n}_{f}
\end{array}\right) \\
& =\left(\begin{array}{c}
\hat{n}_{f} \\
\hat{b}_{f}
\end{array}\right)^{T}\left[\begin{array}{c}
\partial \hat{b}_{f} / \partial \boldsymbol{\omega} \\
{[0]_{3,3}}
\end{array}\right]\left[\begin{array}{c}
\partial \hat{b}_{f} / \partial \mathbf{C} \\
\left.\partial \hat{n}_{f} / \partial \mathbf{C}\right]
\end{array}\right]\left(\begin{array}{l}
\delta \boldsymbol{\omega} \\
\delta \mathbf{C}
\end{array}\right) .
\end{aligned}
$$

Since $\left[\partial \hat{n}_{f} / \partial \mathbf{C}\right]=\left[\partial \hat{n}_{f} / \partial \mathbf{n}_{f}\right]\left[\partial \mathbf{n}_{f} / \partial \mathbf{T}_{f}\right]\left[\partial \mathbf{T}_{f} / \partial \mathbf{C}\right]$ and $\left[\partial \hat{b}_{f} / \partial \mathbf{b}_{f}\right]$ have already been found, so only $\left[\partial \mathbf{b}_{f} / \partial \mathbf{C}\right]$ and $\left[\partial \mathbf{b}_{f} / \partial \boldsymbol{\omega}\right]$ need to be investigated. The first variation in (82) yields 


$$
\begin{aligned}
\delta \mathbf{b}_{f}= & \delta \boldsymbol{a}_{f}-[\boldsymbol{\omega}]^{\times 2}\left(\delta \mathbf{P}_{f}-\delta \mathbf{G}\right) \\
& +\left(\left[\boldsymbol{\omega} \times\left(\mathbf{P}_{f}-\mathbf{G}\right)\right]^{\times}+[\boldsymbol{\omega}]^{\times}\left[\mathbf{P}_{f}-\mathbf{G}\right]^{\times}\right) \delta \boldsymbol{\omega} \\
= & {\left[\left[\boldsymbol{\omega} \times\left(\mathbf{P}_{f}-\mathbf{G}\right)\right]^{\times}+[\boldsymbol{\omega}]^{\times}\left[\mathbf{P}_{f}-\mathbf{G}\right]^{\times},\right.} \\
& {\left[\frac{\partial \boldsymbol{a}_{f}}{\partial \mathbf{C}}\right]+\left[\frac{\partial \boldsymbol{a}_{f}}{\partial \mathbf{P}_{f}}\right]\left[\frac{\partial \mathbf{P}_{f}}{\partial \mathbf{T}_{f}}\right]\left[\frac{\partial \mathbf{T}_{f}}{\partial \mathbf{C}}\right] } \\
& \left.+[\boldsymbol{\omega}]^{\times 2}\left(\left[\frac{\partial \mathbf{G}}{\partial \mathbf{C}}\right]-\left[\frac{\partial \mathbf{P}_{f}}{\partial \mathbf{C}}\right]\right)\right]\left(\begin{array}{l}
\delta \boldsymbol{\omega} \\
\delta \mathbf{C}
\end{array}\right),
\end{aligned}
$$

where $\left[\partial \boldsymbol{a}_{f} / \partial \mathbf{P}_{f}\right]$ denote the gravity gradient matrix of the polyhedron gravity model evaluated at the reference facet center. $\left[\partial \boldsymbol{a}_{f} / \partial \mathbf{C}\right]$ has already been found and also,

$$
\left[\frac{\partial \mathbf{P}_{f}}{\partial \mathbf{C}}\right]=\frac{1}{3}\left[\begin{array}{lll}
{[I]_{3}} & {[I]_{3}} & {[I]_{3}}
\end{array}\right]\left[\frac{\partial \mathbf{T}_{f}}{\partial \mathbf{C}}\right] .
$$

Making the assumption that the body is rotating about an arbitrary axis $\hat{e}$, we have

$$
\omega=\omega \hat{e} .
$$

If $\hat{e}$ is taken as the largest inertia axis, the small body is undergoing principal axis rotation and one can write

$$
\omega=\omega[\mathcal{P B}]^{T} \hat{e}_{3},
$$

where $\mathcal{P}$ and $\mathcal{B}$, respectively, stand for the principal and current body-fixed frames, and $\hat{e}_{3}=\left(\begin{array}{lll}0 & 0 & 1\end{array}\right)^{T}$. Letting $[\mathcal{P B}]$ be parametrized by a set of modified Rodrigues parameters $\left(\mathrm{O}^{\prime}\right.$ Keefe and Schaub 2014) $\sigma$ such that $[\mathcal{P B}]=[\mathcal{P B}](\sigma)$, linearizing a perturbed direction cosine matrix $(\mathrm{DCM})\left[\mathcal{P}^{\prime} \mathcal{B}\right]$ about a reference $[\mathcal{P} \mathcal{B}]$ yields

$$
\left[\mathcal{P}^{\prime} \mathcal{P}\right]=[I]_{3}-4[\delta \sigma]^{\times}
$$

As a result, the first variation in the angular velocity caused by a change in the spin rate and principal axes direction is given by

$$
\begin{aligned}
\delta \omega & =\left[\begin{array}{ll}
{[\mathcal{P B}]^{T} \hat{e}_{3}} & -4 \omega[\mathcal{P B}]^{T}\left[\hat{e}_{3}\right]^{\times}
\end{array}\right]\left(\begin{array}{l}
\delta \omega \\
\delta \boldsymbol{\sigma}
\end{array}\right)=\left[\left(\frac{\partial \omega}{\partial \omega}\right)\left[\frac{\partial \omega}{\partial \boldsymbol{\sigma}}\right]\right]\left(\begin{array}{l}
\delta \omega \\
\delta \boldsymbol{\sigma}
\end{array}\right) \\
& =\left[\left(\frac{\partial \omega}{\partial \omega}\right)\left[\frac{\partial \omega}{\partial \boldsymbol{\sigma}}\right]\right]\left[\begin{array}{cc}
1 & \mathbf{0}_{3 N_{C}}^{T} \\
\mathbf{0}_{3} & {[\partial \boldsymbol{\sigma} / \partial \mathbf{C}]}
\end{array}\right]\left(\begin{array}{l}
\delta \omega \\
\delta \mathbf{C}
\end{array}\right)=\left[\left(\frac{\partial \omega}{\partial \omega}\right)\left[\frac{\partial \omega}{\partial \mathbf{C}}\right]\right]\left(\begin{array}{l}
\delta \omega \\
\delta \mathbf{C}
\end{array}\right) .
\end{aligned}
$$

\subsection{Variance in the slope}

The variance in the slope is thus given by

$$
\sigma_{s}^{2}=[\partial s / \partial \omega(\partial s / \partial \mathbf{C})]\left[\begin{array}{cc}
\sigma_{\omega}^{2} & \boldsymbol{\gamma}_{\mathbf{C}, \omega}^{T} \\
\boldsymbol{\gamma}_{\mathbf{C}, \omega}\left[P_{\mathbf{C C}}\right]
\end{array}\right]\left[\begin{array}{c}
\partial s / \partial \omega \\
(\partial s / \partial \mathbf{C})^{T}
\end{array}\right],
$$

where $\sigma_{\omega}^{2}$ denotes the standard-deviation in the angular velocity of the body, and $\boldsymbol{\gamma}_{\mathbf{C}, \omega}$ the cross-correlation vector between the shape errors and the angular velocity. 


\section{Populating the shape covariance matrix}

\subsection{Gaussian-truncated shape uncertainty}

A straightforward way to set the shape covariance matrix, such that random shapes sampled from this Gaussian distribution present the suitable level of statistical variability, is to populate its partitions according to the following rule, where $\sigma$ and $l$, respectively, denote the standard deviation and correlation length of the surface noise (Bercovici and Mcmahon 2019)

$$
\left[P_{\mathbf{C}_{i}} \mathbf{C}_{i}\right]=\sigma^{2}\left(\hat{n}_{i} \hat{n}_{i}^{T}+\epsilon\left[\hat{e}_{1} \hat{e}_{1}^{T}+\hat{e}_{2} \hat{e}_{2}^{T}\right]\right),
$$

such that $\epsilon<<1, \hat{n}_{i}$ being the averaged outward surface normal at $\mathbf{C}_{i} \cdot \hat{n}_{i}, \hat{e}_{2}$ and $\hat{e}_{3}$ form an orthonormal basis originating at each control point. This effectively makes the uncertainty at each control point greatest along their normal. In addition, the vertices are correlated with each other through the correlation matrix

$$
\left[P_{\mathbf{C}_{i} \mathbf{C}_{j}}\right]=\sigma^{2} \exp \left(-\frac{\left\|\mathbf{C}_{i}-\mathbf{C}_{j}\right\|^{2}}{l^{2}}\right) \hat{n}_{i} \hat{n}_{j}^{T} .
$$

This correlation matrix was automatically set to zero, should $\left\|\mathbf{C}_{i}-\mathbf{C}_{j}\right\|$ become larger than $3 l$.

\subsection{Polar shape uncertainty}

The actual uncertainty in some asteroid shape models is not homogeneously distributed, but concentrated around the poles. This is a common issue in radar astronomy as a number of published asteroid shape models feature poor observability of high latitudes (Scheeres et al. 2006a; Busch et al. 2006, 2011). This paragraph thus explores the application of local uncertainty regions. A local uncertainty region centered at $\mathbf{C}_{c}$ and characterized by a correlation distance $l$ and standard deviation $\sigma$ can be formed by adding the following $3 \times 3$ partition to the corresponding block in $\left[P_{\mathbf{C C}}\right]$ :

$$
\begin{aligned}
{\left[P_{\mathbf{C}_{i} \mathbf{C}_{j}}\right]=} & {\left[P_{\mathbf{C}_{i} \mathbf{C}_{j}}\right]+\sigma^{2} \hat{n}_{i} \hat{n}_{j}^{T} \exp \left(-\frac{1}{2 l^{2}}\left(\left\|\mathbf{C}_{i}-\mathbf{C}_{c}\right\|^{2}\right.\right.} \\
& \left.\left.+\left\|\mathbf{C}_{j}-\mathbf{C}_{c}\right\|^{2}+2\left\|\mathbf{C}_{i}-\mathbf{C}_{j}\right\|^{2}\right)\right)
\end{aligned}
$$

This expression covers all cases, when $i=j$ as well as when $i \neq j$. This covariance matrix partition is set to zero should any of the distances $\left\|\mathbf{C}_{i}-\mathbf{C}_{c}\right\|,\left\|\mathbf{C}_{j}-\mathbf{C}_{c}\right\|$ or $\left\|\mathbf{C}_{i}-\mathbf{C}_{j}\right\|$ become greater than $3 l$.

\subsection{Covariance regularization}

Uncertainty quantification over large shape models by means of the proposed method requires some special care. Although memory availability is not a concern for ground-based computations, numerical errors very much are, as the extraction of the covariance square root by means of a Cholesky decomposition is likely to fail as the covariance matrix grows in size. The spectral decomposition of the covariance used in Bercovici and Mcmahon (2019) has been found to be generally more stable than the Cholesky equivalent, but requires the 

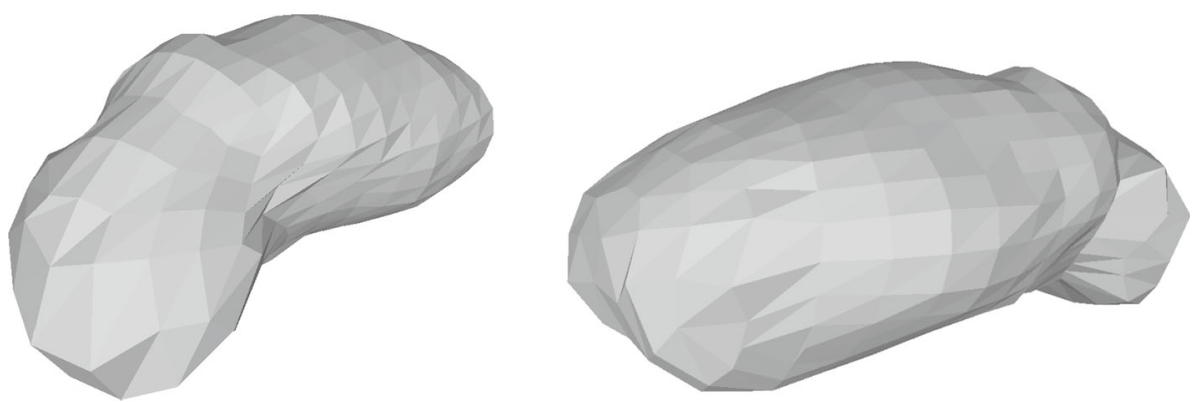

Fig. 225143 Itokawa-8 shape model

covariance matrix to remain positive semi-definite to be usable as is. This positive semidefiniteness can be violated either by construction of the covariance, or by numerical errors that creep-in during the eigenvalue computation process. The shape covariance matrix must thus be investigated before extracting its square root or running it through the linearized uncertainty quantification pipeline, and appropriate measures be taken so as to ensure that it remains positive semi-definite at all times.

A simple yet satisfactory covariance regularization scheme consists in first computing the eigenvalue decomposition of the prescribed vertex covariance $\left[P_{\mathbf{C C}}\right]$ as in

$$
\left[P_{\mathbf{C C}}\right]=[U][D][U]^{T},
$$

where $D$ is the diagonal matrix of eigenvalues, then by regularizing $D$ itself. That is, a new diagonal matrix of real eigenvalues $D^{\prime}$ is defined through

$$
[D]^{\prime}(i, i)=\max (0, \operatorname{Re}([D](i, i))) \forall i \in\left[0 \ldots 3 N_{C}-1\right] .
$$

The zero-clamped eigenvalue matrix $[D]^{\prime}$ can be combined with the original eigenvectors to produce a well-behaved covariance matrix through $\left[P_{\mathbf{C C}}\right]=[U][D]^{\prime}[U]^{T}$ along with the covariance square root $\sqrt{\left[P_{\mathbf{C C}}\right]^{\prime}}=[U] \sqrt{[D]^{\prime}}[U]^{T}$.

\section{Results}

\subsection{Itokawa}

The methods developed in this paper are demonstrated over asteroid 25143 Itokawa, subjected to significant deviations in its vertex coordinates. The reference shape model is shown on Fig. 2. The model used to generate the vertices covariance is similar as in Bercovici and Mcmahon (2019), while the shape model is derived from downsampling the original shape model from Gaskell et al. (2008). The inputs used in the successive simulations are listed on Tables 1 and 2. The shapes drawn from the Monte-Carlo and overlaid with the reference, unperturbed 25143 Itokawa- 8 shape outline can be found on Fig. 3. The smaller correlation distance causes a more erratic behavior of otherwise neighboring vertices.

The proposed uncertainty model enables the evaluation of the variance of the potential $\sigma_{U}^{2}$ and the covariance of the acceleration $\left[P_{\mathbf{a}}\right]$. The present section focuses on the acceleration. The model was first validated by comparing the predicted uncertainty levels to these obtained from Monte-Carlo simulations where shape outcomes were randomly sampled and used to 
Table 1 Inputs to 25143 Itokawa's polyhedron gravity uncertainty model, Cases 1 to 6

\begin{tabular}{lrrrrrr}
\hline & Case 1 & Case 2 & Case 3 & Case 4 & Case 5 & Case 6 \\
\hline Correlation distance $(\mathrm{m})$ & 100 & 100 & 100 & 200 & 200 & 200 \\
Standard deviation in normal error $(\mathrm{m})$ & 10 & 10 & 10 & 10 & 10 & 10 \\
Monte-Carlo samples & 300 & 1000 & 3000 & 300 & 1000 & 3000 \\
\hline
\end{tabular}

Fig. 330 outcomes from the Monte-Carlo sampling of the shape deviations (lightblue), overlaid with the reference 25143 Itokawa-8 shape model (black), (Cases 1, 2 and 3)

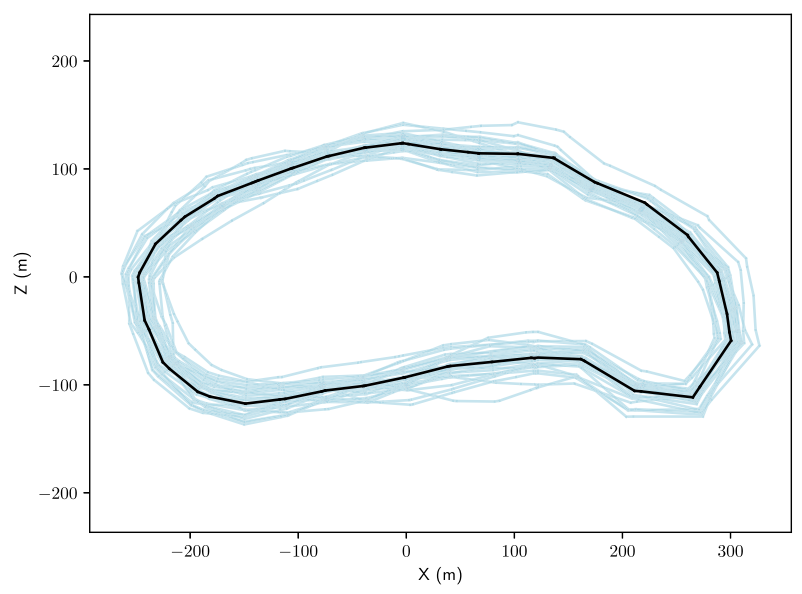

Table 2 Inputs to 25143 Itokawa's polyhedron gravity uncertainty model, Cases 7 to 9

\begin{tabular}{lrrr}
\hline & Case 7 & Case 8 & Case 9 \\
\hline Correlation distance $(\mathrm{m})$ & 250 & 250 & 250 \\
Standard deviation in normal error $(\mathrm{m})$ & 20 & 20 & 20 \\
Monte-Carlo samples & 300 & 1000 & 3000 \\
\hline
\end{tabular}

evaluate acceleration outcomes at selected positions. Such selected field points were picked such that they never encroach the inside of the randomly sampled Monte-Carlo shapes. The justification for this lies in the discontinuity of the performance factors $\Omega_{f}$ across the surface. As noted by Werner (1997), they correspond to the signed solid angle protracted by a facet element as seen from a field point. As such, the sign of $\Omega_{f}$ flips as the field point moves across the surface of the shape. It is thus inevitable that the first-order acceleration uncertainty model will not match the statistics of a Monte-Carlo distribution featuring accelerations calculated at field points that may encroach the stochastic shapes (Fig. 4).

The acceleration covariance at the selected field point $\left[P_{\mathbf{a}}\right]$ was compared to the one computed from the MC acceleration distribution so as to assess the accuracy of the uncertainty model relative to the Monte-Carlo runs, as in percentages of $\|\left[P_{\mathbf{a}}\right]-$ $\left[P_{\mathbf{a}}^{\mathrm{MC}}\right] \|_{2} /$ trace $\left(\left[P_{\mathbf{a}}^{\mathrm{MC}}\right]\right)$ where $\left[P_{\mathbf{a}}^{\mathrm{MC}}\right]$ denotes the covariance in the Monte-Carlo acceleration outcomes and $\|\cdot\|_{2}$ the usual L2-norm.

The agreement between the predicted covariance and the Monte-Carlo one measured by the L2 criterion is shown on Figs. 7 and 8. Field point colored in gray denote locations that have encroached the Monte-Carlo shapes and that are thus excluded from the statistics. The distribution of the accuracy metric over all the field points that never the shape is shown in 
Fig. 430 outcomes from the Monte-Carlo sampling of the shape deviations (lightblue), overlaid with the reference 25143 Itokawa-8 shape model (black), (Case 7, 8 and 9). Unlike the other cases, the MC shapes are sometimes self-intersecting

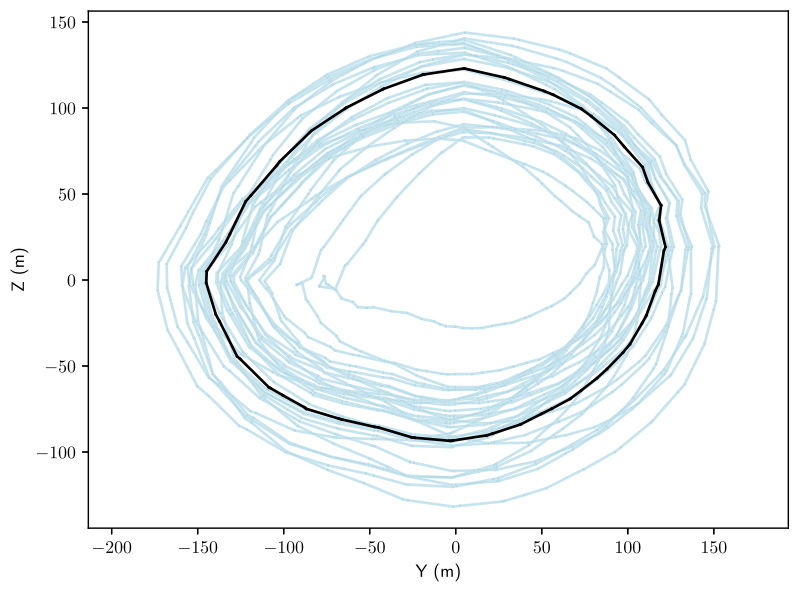

Figs. 5 and 6, in addition to flagging the field points that have encroached the Monte-Carlo shapes.

Two important trends are noticeable: first, the number of shape samples considered in the Monte-Carlo analysis plays a dominant role in the agreement with the proposed linearized approach. For Cases 1 through 6, the mutual agreement between the MC acceleration dispersion and the linearized prediction improves as more shape samples are added. This shows that the linearized model is capable of capturing most of the uncertainty in the acceleration arising from the shape uncertainty, at a lesser cost than a Monte-Carlo sampling of the shape providing the same level of accuracy, while remaining consistent with larger-sample-size statistics. Second, the agreement between the two statistical moments appears to be best away from the shape. Our method remains consistent within the Brillouin sphere, almost all the way to the surface of the shape. Outliers showing in the large-sample-size MC Cases 3 and 6 appear close to the shape.

Figure 4 depicts a few Y-Z slices of the sampled shapes using $20 \mathrm{~m}$ of surface noise standard deviations and $250 \mathrm{~m}$ of correlation length. The resulting stochastic shapes present considerable differences in size relative to the reference shape model about which the polyhedron gravity model is linearized. It is worth noting that surface self-intersections can be seen, indicative that the resolution of the shape model is too high relative to the amount of shape uncertainty being considered. Nevertheless, these cases serve as a stress test for our linearized gravity uncertainty model. As can be seen on Fig. 9, Cases 7, 8 and 9 present commensurable larger errors between the linear uncertainty model and the MC statistics, that do not exceed $14 \%$ in Case 9 . It thus possible to conclude that despite the spuriously large amount of statistical uncertainty in the shape-the dimension and resolution of the reference shape model are not consistent with the amount of surface noise, as noted earlier- the linear uncertainty model has not completely broken down.

The predictive aspect of the gravity uncertainty model is shown on Figs. 10 and 11, that depict the relative uncertainty in the acceleration predicted by the model, measured in percentages of $\sqrt{\operatorname{trace}\left(\left[P_{\mathbf{a}}\right]\right)} /\|\mathbf{a}\|$. These maps were obtained by sampling three uniform $10-\mathrm{m}$ spaced, orthogonal planar grids. It can be seen that the uncertainty in the acceleration is greatest close to the shape, and decreases as the distance to the surface increases. Moreover, the impact of the correlation length is apparent in the uncertainty levels. Increasing the correlation lengths causes the vertices deviations to interact in a constructive manner, effectively 
Relative accuracy $\left(\left\|\left[P_{\mathbf{a}}\right]-\left[P_{\mathbf{a}}^{\mathrm{MC}}\right]\right\|_{2} / \operatorname{trace}\left(\left[P_{\mathbf{a}}^{\mathrm{MC}}\right]\right) \cdot 100 \%\right)$

\begin{tabular}{|c|c|c|c|c|c|c|c|c|c|c|c|c|}
\hline & $X(m)$ & $\mathrm{Y}(\mathrm{m})$ & $\mathrm{Z}(\mathrm{m})$ & Case 1 & Case 2 & Case 3 & Case 4 & Case 5 & Case 6 & Case 7 & Case 8 & Case 9 \\
\hline 1 & 0.0 & -480.0 & 0.0 & 1.48 & 3.68 & 6.29 & 5.17 & 0.78 & 3.35 & 6.21 & 5.22 & 8.91 \\
\hline 2 & 0.0 & -400.0 & 0.0 & 2.35 & 4.47 & 6.61 & 5.23 & 0.65 & 3.48 & 7.01 & 5.68 & 9.17 \\
\hline 3 & -339.41 & -339.41 & 0.0 & 8.72 & 1.3 & 4.14 & 9.85 & 2.36 & 2.27 & 2.2 & 2.62 & 7.73 \\
\hline 4 & 0.0 & -339.41 & -339.41 & 1.98 & 3.03 & 5.82 & 7.41 & 1.39 & 3.26 & 4.82 & 4.77 & 8.36 \\
\hline 5 & 0.0 & -339.41 & 339.41 & 2.46 & 2.35 & 5.45 & 3.12 & 0.91 & 3.01 & 6.96 & 4.07 & 8.37 \\
\hline 6 & 339.41 & -339.41 & 0.0 & 1.0 & 4.97 & 5.78 & 1.36 & 0.77 & 3.94 & 8.85 & 8.69 & 10.34 \\
\hline 7 & 0.0 & -320.0 & 0.0 & 4.09 & 5.6 & 6.98 & 5.08 & 1.06 & 3.69 & 8.42 & 6.46 & 9.65 \\
\hline 8 & -282.84 & -282.84 & 0.0 & 10.32 & 1.4 & 4.11 & 10.34 & 2.25 & 2.37 & 3.13 & 2.92 & 8.04 \\
\hline 9 & 0.0 & -282.84 & -282.84 & 1.61 & 3.58 & 6.08 & 8.1 & 1.35 & 3.31 & 5.2 & 4.98 & 8.44 \\
\hline 10 & 0.0 & -282.84 & 282.84 & 3.46 & 2.86 & 5.66 & 2.91 & 0.72 & 3.1 & 7.72 & 4.27 & 8.44 \\
\hline 11 & 282.84 & -282.84 & 0.0 & 1.46 & 5.46 & 5.71 & 1.73 & 0.99 & 4.01 & 9.18 & 9.46 & 10.56 \\
\hline 12 & 0.0 & -240.0 & 0.0 & 7.42 & 7.06 & 7.19 & 4.28 & 2.26 & 4.09 & 11.34 & 8.12 & 10.79 \\
\hline 13 & -226.27 & -226.27 & 0.0 & 11.69 & 1.41 & 4.04 & 10.49 & 1.93 & 2.67 & 4.15 & 3.65 & 8.73 \\
\hline 14 & 0.0 & -226.27 & -226.27 & 2.43 & 4.36 & 6.42 & 9.01 & 1.27 & 3.36 & 5.9 & 5.3 & 8.62 \\
\hline 15 & 0.0 & -226.27 & 226.27 & 5.04 & 3.59 & 5.91 & 2.53 & 1.01 & 3.25 & 8.88 & 4.57 & 8.52 \\
\hline 16 & 226.27 & -226.27 & 0.0 & 2.15 & 5.76 & 5.49 & 2.22 & 1.28 & 4.04 & 9.26 & 10.0 & 10.51 \\
\hline 17 & -169.71 & -169.71 & 0.0 & 9.09 & 2.21 & 3.91 & 9.74 & 1.96 & 3.2 & inside & inside & inside \\
\hline 18 & 0.0 & -169.71 & -169.71 & 4.74 & 5.34 & 6.83 & 10.15 & 1.13 & 3.39 & 7.36 & 5.96 & 9.1 \\
\hline 19 & 0.0 & -169.71 & 169.71 & 7.33 & 4.6 & 6.15 & 1.81 & 1.71 & 3.51 & 10.71 & 5.03 & 8.55 \\
\hline 20 & 169.71 & -169.71 & 0.0 & 2.95 & 5.75 & 5.37 & 2.74 & 1.71 & 4.05 & 9.19 & 9.04 & 9.73 \\
\hline 21 & 0.0 & -113.14 & -113.14 & 8.25 & 6.04 & 7.32 & 11.28 & 1.44 & 3.31 & inside & inside & inside \\
\hline 22 & 0.0 & -113.14 & 113.14 & 9.51 & 5.51 & 6.35 & 2.51 & inside & inside & inside & inside & inside \\
\hline 23 & -600.0 & 0.0 & 0.0 & 5.72 & 1.24 & 1.92 & 10.23 & 3.23 & 1.46 & 1.98 & 1.13 & 6.22 \\
\hline 24 & -500.0 & 0.0 & 0.0 & 6.19 & 1.08 & 1.61 & 10.73 & 3.37 & 1.4 & 3.14 & 1.12 & 6.11 \\
\hline 25 & -424.26 & 0.0 & -424.26 & 5.42 & 1.21 & 3.04 & 11.07 & 3.03 & 1.98 & 1.51 & 1.69 & 6.66 \\
\hline 26 & -424.26 & 0.0 & 424.26 & 3.86 & 1.93 & 2.33 & 6.97 & 2.37 & 1.48 & 2.34 & 0.95 & 6.27 \\
\hline 27 & -400.0 & 0.0 & 0.0 & 6.01 & 0.65 & 1.5 & 10.65 & 3.44 & 1.49 & 4.61 & 1.46 & 6.08 \\
\hline 28 & -353.55 & 0.0 & -353.55 & 6.48 & 1.56 & 2.81 & 12.38 & 3.2 & 1.9 & 2.5 & 1.5 & 6.58 \\
\hline 29 & -353.55 & 0.0 & 353.55 & 4.49 & 2.36 & 2.02 & 7.24 & 2.3 & 1.3 & 2.32 & 0.7 & 5.99 \\
\hline 30 & -300.0 & 0.0 & 0.0 & 3.82 & 3.1 & 2.9 & 8.14 & 3.15 & 2.2 & 6.15 & inside & inside \\
\hline 31 & -282.84 & 0.0 & -282.84 & 7.99 & 2.19 & 2.49 & 14.18 & 3.33 & 1.84 & 3.98 & 1.27 & 6.57 \\
\hline 32 & -282.84 & 0.0 & 282.84 & 5.23 & 2.95 & 1.7 & 7.45 & 2.05 & 1.08 & 2.46 & 0.59 & 5.59 \\
\hline 33 & -212.13 & 0.0 & -212.13 & 10.08 & 3.39 & 2.02 & 16.66 & 3.14 & 1.89 & 6.1 & 1.14 & 6.92 \\
\hline 34 & -212.13 & 0.0 & 212.13 & 5.81 & 3.67 & 1.54 & 7.47 & 1.4 & 0.9 & 2.98 & 1.05 & 5.0 \\
\hline 35 & -141.42 & 0.0 & -141.42 & 10.35 & inside & inside & 19.02 & inside & inside & inside & inside & inside \\
\hline 36 & -141.42 & 0.0 & 141.42 & 5.51 & 3.32 & 1.88 & 7.01 & 1.19 & 1.27 & inside & inside & inside \\
\hline 37 & 0.0 & 0.0 & -480.0 & 1.69 & 1.95 & \begin{tabular}{ll|}
4.47 \\
\end{tabular} & 7.27 & 1.75 & 2.92 & 4.61 & 3.86 & 7.36 \\
\hline 38 & 0.0 & 0.0 & -400.0 & 2.2 & 2.34 & 4.49 & 7.96 & 1.83 & 2.89 & 4.85 & 3.9 & 7.19 \\
\hline 39 & 0.0 & 0.0 & -320.0 & 3.2 & 2.99 & 4.53 & 8.89 & 1.93 & 2.82 & 5.27 & 3.97 & 6.98 \\
\hline 40 & 0.0 & 0.0 & -240.0 & 5.03 & 4.15 & 4.69 & 10.0 & 1.97 & 2.68 & 6.14 & 4.21 & 6.81 \\
\hline 41 & 0.0 & 0.0 & -160.0 & 8.31 & 6.43 & 5.34 & 10.45 & 1.58 & 2.57 & 8.8 & 5.46 & inside \\
\hline
\end{tabular}

Fig. 5 Relative accuracy percentage at selected points. The first three columns are the coordinates of the field point with respect to the 25143 Itokawa 8 shape model axes 
Relative accuracy $\left(\left\|\left[P_{\mathbf{a}}\right]-\left[P_{\mathbf{a}}^{\mathrm{MC}}\right]\right\|_{2} / \operatorname{trace}\left(\left[P_{\mathbf{a}}^{\mathrm{MC}}\right]\right) \cdot 100 \%\right)$

\begin{tabular}{|c|c|c|c|c|c|c|c|c|c|c|c|c|}
\hline & $X(m)$ & $Y(m)$ & $\mathrm{Z}(\mathrm{m})$ & Case 1 & Case 2 & Case 3 & Case 4 & Case 5 & Case 6 & Case 7 & Case 8 & Case 9 \\
\hline 42 & 0.0 & 0.0 & 160.0 & 9.73 & 2.05 & inside & 2.61 & 1.8 & 3.31 & inside & inside & inside \\
\hline 43 & 0.0 & 0.0 & 240.0 & 6.86 & 2.26 & 3.55 & 3.14 & 1.33 & 2.58 & 9.34 & 2.13 & 7.61 \\
\hline 44 & 0.0 & 0.0 & 320.0 & 5.25 & 2.03 & 3.7 & 3.08 & 1.42 & 2.49 & 8.07 & 2.23 & 7.48 \\
\hline 45 & 0.0 & 0.0 & 400.0 & 4.06 & 1.7 & 3.79 & 3.01 & 1.48 & 2.47 & 7.27 & 2.32 & 7.49 \\
\hline 46 & 0.0 & 0.0 & 480.0 & 3.22 & 1.41 & 3.85 & 2.96 & 1.5 & 2.47 & 6.73 & 2.42 & 7.52 \\
\hline 47 & 141.42 & 0.0 & -141.42 & 6.35 & 4.9 & 3.31 & 2.9 & 1.54 & 2.02 & 6.19 & 6.21 & 4.72 \\
\hline 48 & 141.42 & 0.0 & 141.42 & 7.63 & 4.12 & inside & 6.3 & 3.95 & 5.3 & inside & inside & inside \\
\hline 49 & 212.13 & 0.0 & -212.13 & 3.69 & 4.44 & 4.2 & 2.13 & 1.59 & 3.57 & 12.3 & 11.83 & 10.79 \\
\hline 50 & 212.13 & 0.0 & 212.13 & 5.21 & 2.71 & 5.52 & 5.04 & 1.96 & 3.86 & 10.89 & 6.68 & 10.65 \\
\hline 51 & 282.84 & 0.0 & -282.84 & 2.84 & 4.18 & 4.62 & 1.57 & 1.34 & 3.89 & 11.47 & 10.65 & 10.91 \\
\hline 52 & 282.84 & 0.0 & 282.84 & 4.3 & 2.68 & 5.3 & 3.71 & 1.25 & 3.68 & 9.86 & 6.42 & 10.05 \\
\hline 53 & 353.55 & 0.0 & -353.55 & 2.09 & 3.75 & 4.83 & 1.27 & 0.99 & 3.86 & 9.98 & 9.03 & 10.28 \\
\hline 54 & 353.55 & 0.0 & 353.55 & 3.54 & 2.45 & 5.16 & 2.67 & 0.83 & 3.57 & 9.19 & 6.05 & 9.7 \\
\hline 55 & 400.0 & 0.0 & 0.0 & 2.55 & 6.45 & 5.22 & 1.63 & 0.94 & 3.53 & 13.84 & 14.5 & 13.88 \\
\hline 56 & 424.26 & 0.0 & -424.26 & 1.65 & 3.35 & 4.92 & 1.41 & 0.71 & 3.78 & 8.96 & 7.91 & 9.82 \\
\hline 57 & 424.26 & 0.0 & 424.26 & 2.96 & 2.22 & 5.06 & 1.88 & 0.56 & 3.49 & 8.64 & 5.7 & 9.44 \\
\hline 58 & 500.0 & 0.0 & 0.0 & 1.7 & 4.88 & 5.14 & 1.99 & 1.09 & 3.81 & 11.75 & 11.45 & 12.25 \\
\hline 59 & 600.0 & 0.0 & 0.0 & 1.48 & 4.05 & 5.15 & 1.78 & 0.93 & 3.84 & 10.46 & 9.59 & 11.24 \\
\hline 60 & 0.0 & 113.14 & -113.14 & 7.61 & 3.37 & 2.62 & 8.37 & 1.77 & 1.83 & inside & inside & inside \\
\hline 61 & 0.0 & 113.14 & 113.14 & 9.68 & 3.17 & 2.38 & 6.62 & 2.8 & 1.63 & inside & inside & inside \\
\hline 62 & 0.0 & 160.0 & 0.0 & 5.71 & 4.15 & 2.57 & 6.05 & 1.1 & 1.61 & inside & inside & inside \\
\hline 63 & -169.71 & 169.71 & 0.0 & inside & inside & inside & 5.97 & 1.48 & inside & inside & inside & inside \\
\hline 64 & 0.0 & 169.71 & -169.71 & 5.62 & 2.05 & 2.61 & 7.86 & 1.97 & 2.15 & 6.02 & 2.88 & 5.45 \\
\hline 65 & 0.0 & 169.71 & 169.71 & 6.81 & 1.16 & 1.76 & 5.26 & 2.17 & 1.76 & 8.11 & 1.36 & 6.42 \\
\hline 66 & 169.71 & 169.71 & 0.0 & 4.13 & 2.75 & 3.67 & 5.88 & 3.15 & 3.64 & 9.44 & 6.28 & inside \\
\hline 67 & -226.27 & 226.27 & 0.0 & 1.61 & 2.13 & 0.73 & 8.29 & 1.59 & 0.95 & 2.76 & 2.7 & 5.3 \\
\hline 68 & 0.0 & 226.27 & -226.27 & 4.52 & 1.48 & 2.84 & 6.99 & 1.94 & 2.34 & 5.58 & 2.87 & 5.91 \\
\hline 69 & 0.0 & 226.27 & 226.27 & 5.39 & 1.48 & 2.05 & 4.45 & 1.97 & 1.9 & 7.14 & 1.43 & 6.46 \\
\hline 70 & 226.27 & 226.27 & 0.0 & 4.4 & 1.33 & 4.03 & 4.64 & 2.33 & 3.81 & 9.9 & 6.54 & 9.65 \\
\hline 71 & 0.0 & 240.0 & 0.0 & 5.33 & 1.19 & 1.65 & 6.04 & 1.53 & 1.84 & 7.15 & 1.93 & 5.63 \\
\hline 72 & -282.84 & 282.84 & 0.0 & 2.29 & 0.84 & 0.53 & 8.94 & 2.37 & 0.92 & 2.17 & 1.57 & 5.16 \\
\hline 73 & 0.0 & 282.84 & -282.84 & 3.74 & 1.21 & 3.05 & 6.31 & 1.86 & 2.45 & 5.31 & 2.91 & 6.3 \\
\hline 74 & 0.0 & 282.84 & 282.84 & 4.48 & 1.45 & 2.35 & 3.96 & 1.88 & 2.02 & 6.57 & 1.57 & 6.6 \\
\hline 75 & 282.84 & 282.84 & 0.0 & 4.38 & 1.47 & 4.18 & 3.57 & 1.68 & 3.75 & 9.73 & 6.46 & 9.55 \\
\hline 76 & 0.0 & 320.0 & 0.0 & 4.88 & 0.54 & 1.85 & 5.4 & 1.74 & 1.98 & 6.34 & 1.9 & 5.81 \\
\hline 77 & -339.41 & 339.41 & 0.0 & 2.33 & 0.48 & 0.74 & 8.86 & 2.65 & 1.07 & 2.03 & 1.21 & 5.36 \\
\hline 78 & 0.0 & 339.41 & -339.41 & 3.14 & 1.05 & 3.22 & 5.83 & 1.79 & 2.53 & 5.12 & 2.96 & 6.59 \\
\hline 79 & 0.0 & 339.41 & 339.41 & 3.81 & 1.31 & 2.59 & 3.66 & 1.81 & 2.11 & 6.2 & 1.73 & 6.74 \\
\hline 80 & 339.41 & 339.41 & 0.0 & 4.12 & 1.57 & 4.26 & 2.75 & 1.22 & 3.66 & 9.32 & 6.17 & 9.36 \\
\hline 81 & 0.0 & 400.0 & 0.0 & 4.38 & 0.44 & 2.17 & 4.88 & 1.77 & 2.1 & 5.95 & 2.01 & 6.11 \\
\hline 82 & 0.0 & 480.0 & 0.0 & 3.89 & 0.42 & 2.43 & 4.52 & 1.76 & 2.2 & 5.7 & 2.13 & 6.36 \\
\hline
\end{tabular}

Fig. 6 Relative accuracy percentage at selected points, continued. The first three columns are the coordinates of the field point with respect to the 25143 Itokawa 8 shape model axes 
Fig. 7 Accuracy of predicted uncertainty in acceleration relative to Monte Carlo covariance, Case 3

Fig. 8 Accuracy of predicted uncertainty in acceleration relative to Monte Carlo covariance, Case 6

Fig. 9 Accuracy of predicted uncertainty in acceleration relative to Monte Carlo covariance, Case 9
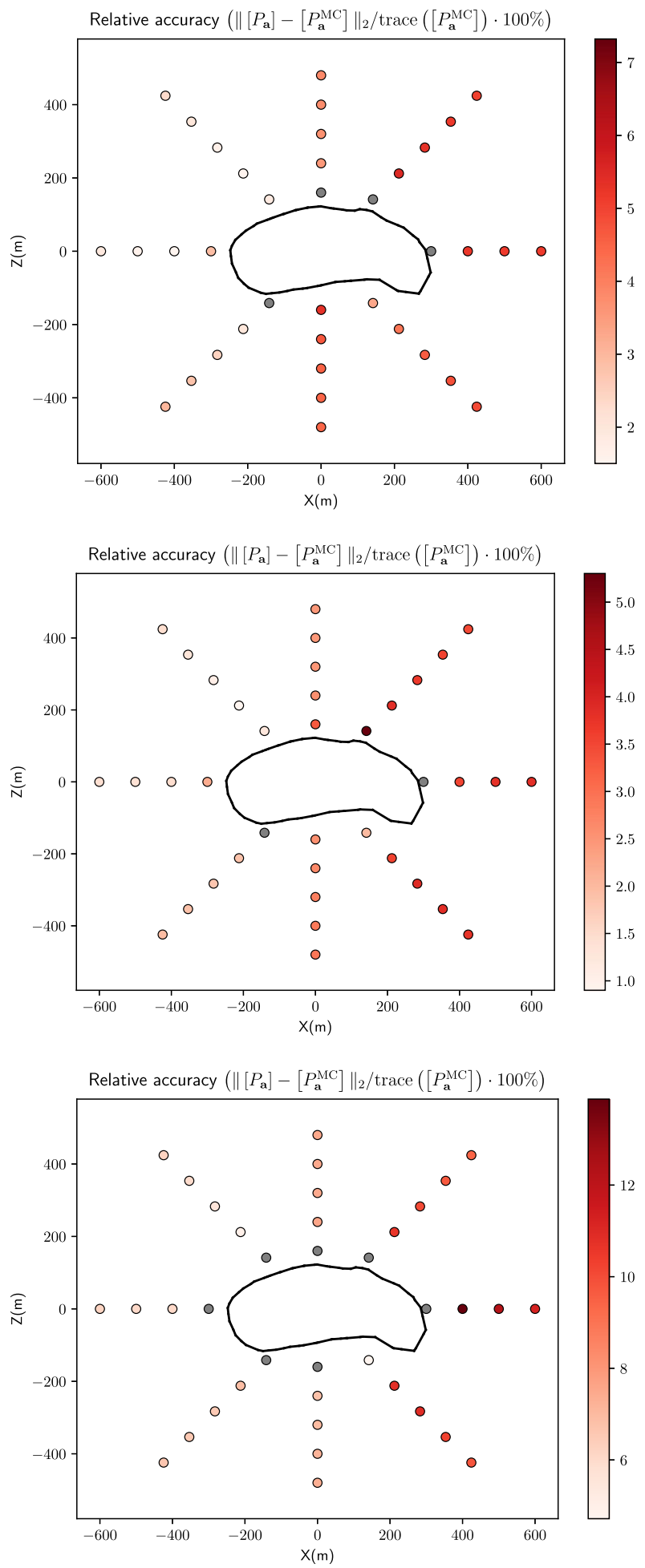
Fig. 10 Relative uncertainty contours around 25143 Itokawa-8, with the inside of BenSecondRevEdit25143 Itokawa shown in white (Case 1, 2 and 3 )

Fig. 11 Relative uncertainty contours around 25143 Itokawa-8, with the inside of 25143 Itokawa shown in white (Case 4, 5 and 6)
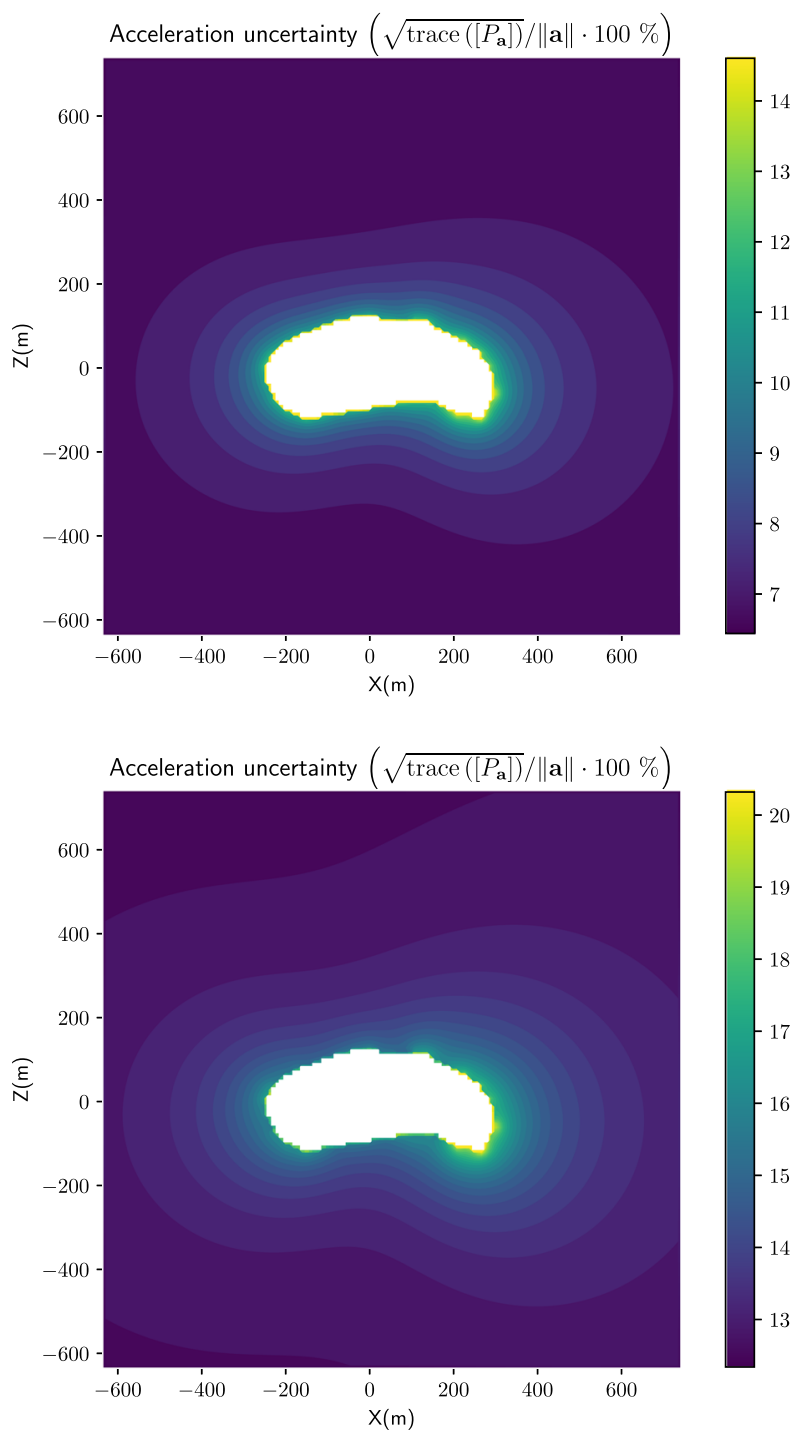

contributing to increasing the volume (hence the mass) uncertainty. It can be seen in all cases that the decay in the uncertainty is initially rapid upon leaving the shape, but becomes much slower as the distance increases.

\subsection{Psyche}

The slope uncertainty model is now demonstrated over the surface of asteroid 16 Psyche, the target of the eponymous upcoming NASA mission. Psyche is thought to be the remnant of a protoplanet core and exhibits a visible/near-infrared characteristic of metal-rich Mclass asteroids (Tedesco et al. 2002). The shape model of Psyche shown on Fig. 12 was reconstructed based on radar observations collected by the Arecibo radio telescope, the only 

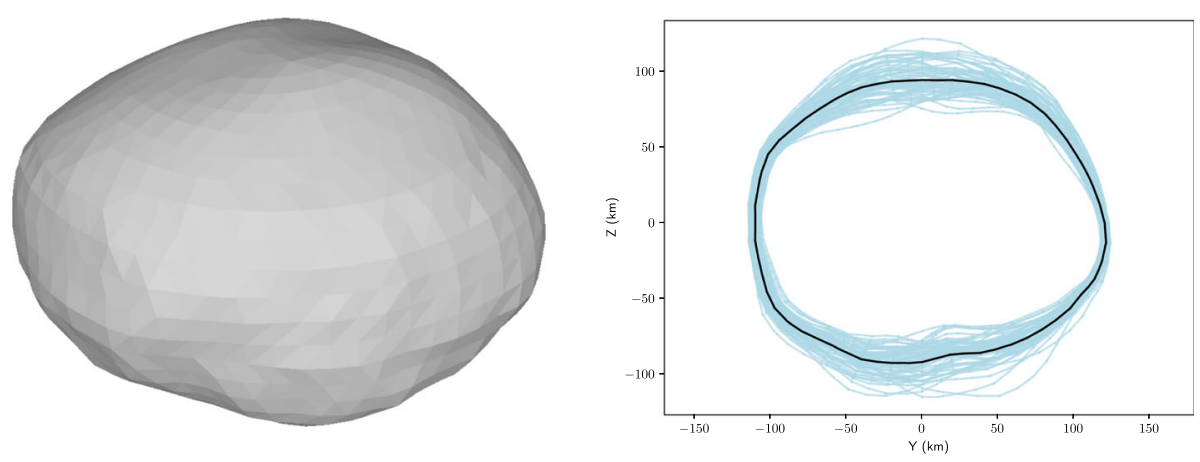

Fig. 12 Left: 16 Psyche shape model. Right: 60 Monte-Carlo shape outcomes (lightblue) overlaid over the reference 16 Psyche shape model (black)

facility on Earth capable of collecting radar observations of main-belt objects (Shepard et al. 2017). The shape model bounding-box spans 279, 232 and $189 \mathrm{~km}$ along its axes of least, intermediate and largest inertia, respectively. The knowledge of Psyche is marked by fairly large uncertainties in the dimensions, density and pole directions, and thus represents an interesting test subject for the proposed gravity uncertainty model. The lack of information on the dynamical environment about Psyche is a strong mission design driver, and stable orbits are typically preferred over other trajectories that may be more fruitful from a science standpoint (Oh et al. 2017; Oguri et al. 2019). Being able to quantify gravity uncertainties arising from the shape would help relax the mission design by providing more insight into the expected variability in the small body dynamical environment.

Inertial acceleration uncertainty The proposed method is employed to define two polar uncertainty regions on Psyche, at -90 and 90 degrees of latitude, centered about vertices 0 and 1147 , respectively in the reference shape model. Both regions feature the same noise standard deviation and correlation length, respectively, set to $10 \mathrm{~km}$ and $75 \mathrm{~km}$. The standard deviation is comparable to the level of uncertainty in the shape reported in Shepard et al. (2017). 5000 Monte-Carlo shapes were drawn to construct the acceleration covariances and means to compare against the analytical prediction of the gravity uncertainty. The bulk density of the asteroid was set to $4500 \mathrm{~kg} / \mathrm{m}^{3}$. A handful of the corresponding shape outcomes from the Monte-Carlo are shown on the right of Fig. 12. The validation of the uncertainty model in the X-Y plane is demonstrated on Fig. 13, as the worst L2-norm covariance error is around $5 \%$. The actual prediction in the gravity uncertainty in the $\mathrm{X}-\mathrm{Y}$ plane is shown on Fig. 14. The structure of the acceleration uncertainty map closely matches that of the underlying shape error, with a maximum uncertainty over the polar regions, but also features a constant background uncertainty of about $4 \%$ all around the body.

Inertial acceleration uncertainty, constant mass The same case as in the previous paragraph was run, but this time with the additional constraint that the mass of Psyche must remain constant as its shape varies. That is, $M=\rho V$ must be conserved. This can only happen if the first variation in the density satisfies $\delta \rho=-\delta V \rho / V$. The partials of the potential and acceleration were thus simply augmented with $-\frac{U(\mathbf{r}) \rho}{V}\left(\frac{\partial V}{\partial \mathbf{C}}\right)$ and $-\frac{\mathbf{a}(\mathbf{r}) \rho}{V}\left(\frac{\partial V}{\partial \mathbf{C}}\right)$ to account for this constraint, where the potential and acceleration are evaluated at the reference density. The analytical prediction in the uncertainty can be found on Fig. 15. The major difference with the previous case lies in the rapid decay in the uncertainty as the queried point moves further away from the shape. The gravity field structure converges towards that of a point-mass as the point moves further away from the shape. Because the standard gravitational parameter 
Fig. 13 L2 Relative accuracy error of analytical gravity uncertainty prediction

Fig. 14 Prediction of gravity acceleration uncertainty around 16 Psyche
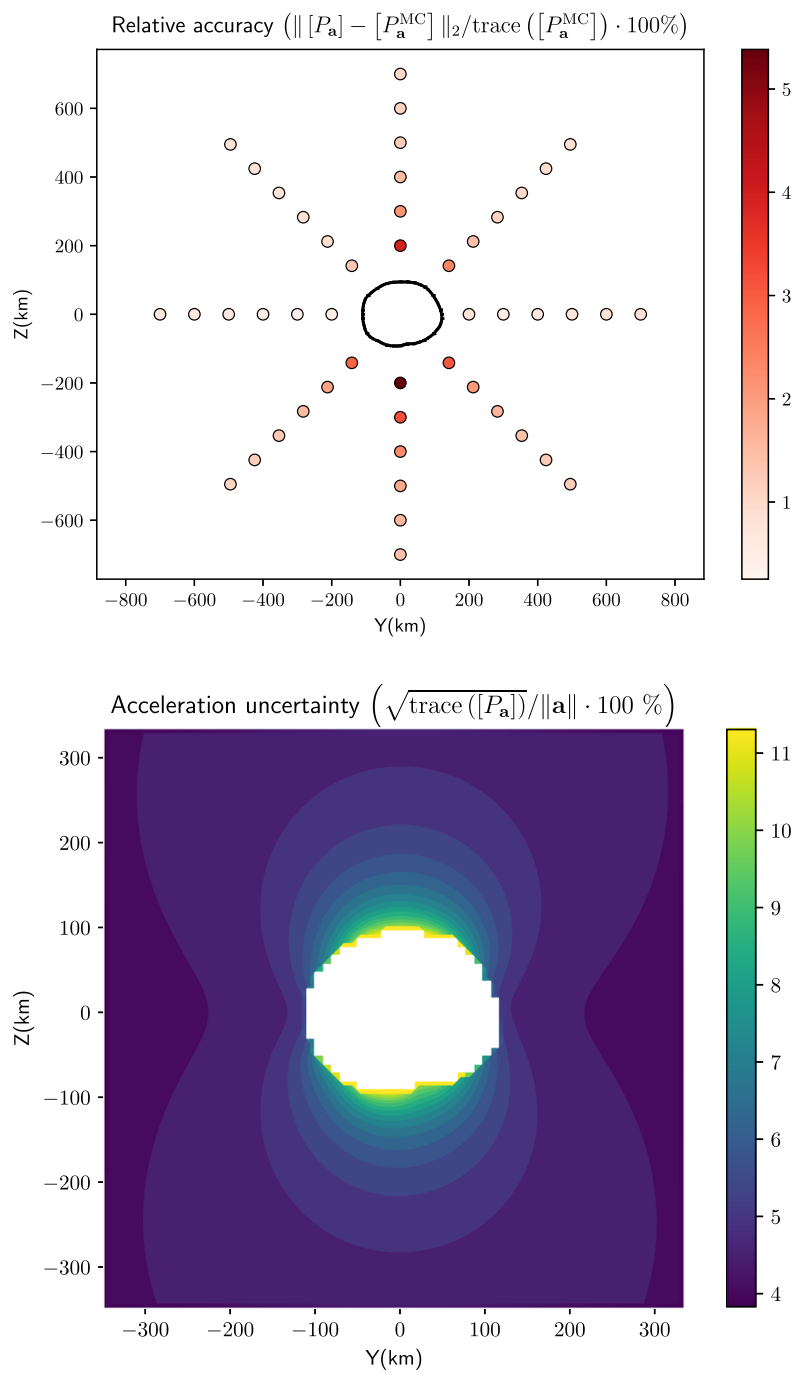

of the shape is constant, there is thus no uncertainty left in the point-mass gravity, causing the acceleration covariance to nullify away from the shape.

Surface slopes The PGM uncertainty model can also applied to the quantification of the uncertainties in the surface slopes of Psyche. The rotation period of Psyche was set to $15105.4 \mathrm{~s}$, in accordance with the reported estimate in Shepard et al. (2017). This reference states that the rotation period is known within $\pm 0.000001 \mathrm{~h}$, which led to considering no uncertainty in the angular velocity magnitude, thus setting $\sigma_{\omega}^{2}=0 \mathrm{rad}^{2} / \mathrm{s}^{2}$ and $\gamma_{\mathbf{C}, \omega}=\mathbf{0}_{3} \mathrm{~m} \cdot \mathrm{rad} / \mathrm{s}$. The two polar uncertainty regions are, however, sufficient to induce local and global uncertainties in the slope, by, respectively, affecting the facet normal and facet body-fixed acceleration.

Before asserting the performance of the prediction in the slope uncertainty, it is necessary to apply a transformation to the predicted slope distribution moments coming out of the developed model. Essentially, this model is oblivious to the fact that slopes are valued between 0 and 180 degrees by construction. In the case of the present paper, this folding issue manifests 
Fig. 15 Prediction in the acceleration uncertainty around 16 Psyche when enforcing constant mass

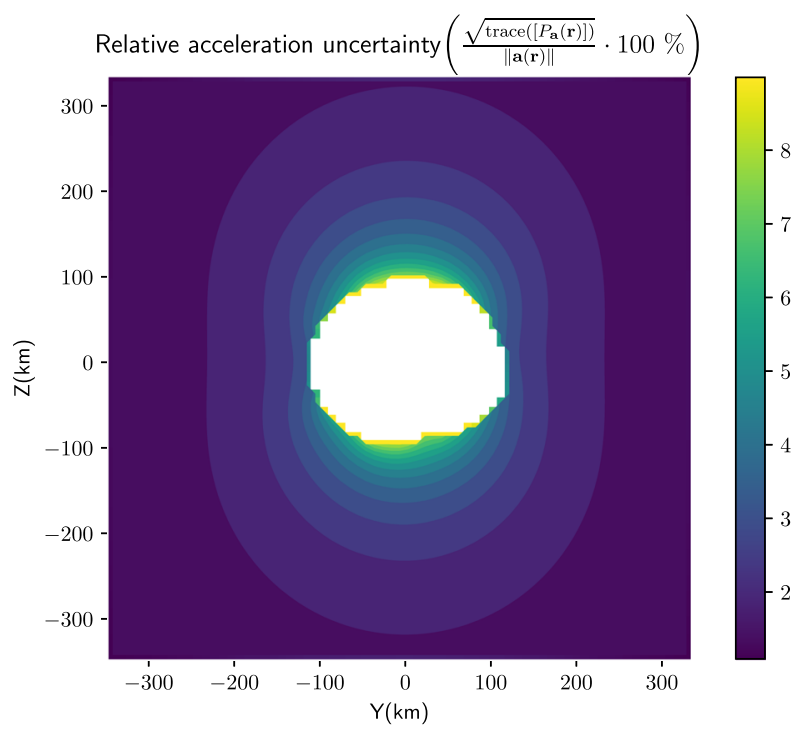

itself in facets whose reference slopes are close to zero. Fortunately, it can be easily remedied by applying the following transform to the unfolded moments $\left(\bar{s}, \sigma_{s}\right)$

$$
\begin{aligned}
\bar{s}_{\text {folded }} & =\sigma_{s} \sqrt{\frac{2}{\pi}} \exp \left(-\bar{s}^{2} /\left(2 \sigma_{s}^{2}\right)\right)+\bar{s} \cdot \operatorname{erf}\left(\bar{s} / \sqrt{2 \sigma_{s}^{2}}\right), \\
\sigma_{s, \text { folded }}^{2} & =\bar{s}^{2}+\sigma_{s}^{2}-\bar{s}_{\text {folded }}^{2} .
\end{aligned}
$$

$\bar{s}$ is nothing else but the slope at the current facet, evaluated using the a-priori shape model. $\left(\bar{s}_{\text {folded }}, \sigma_{s}\right.$,folded $)$ are the mean and second moment about the mean of the distribution obtained from folding the Gaussian of corresponding moments $\left(\bar{s}, \sigma_{s}\right)$ about zero. Note that if $\bar{s}$ is sufficiently far from zero and $\sigma_{s}$ is sufficiently small, then the folding transform leaves the original moments unchanged. Finally, it is important to bear in mind that the proposed folding transform only acts upon the folding that occurs around zero, and does nothing to account for the folding that occurs around 180 degrees. This issue may need to be remedied by a different folding transform, but is not needed here since no slope-reference or perturbedis greater than 90 degrees. The statistics of the zero-folded slopes distribution at two sample facets (facet 0 and facet 1500) were computed and compared to the analytical predictions arising from the linearized acceleration uncertainty model. Table 3 provides the Cartesian coordinates of the facet centers as well as the surface slopes evaluated at the facet centers of the reference shape. The resulting statistics in the slopes distribution and prediction errors are shown on Table 4. Facet 0 lies at the center of one of the two uncertainty regions and is thus highly perturbed by the resulting terrain motion. On the contrary, facet 1500 is much closer to the equator, such that the local shape uncertainty at this facet's center is limited. The slightly degraded accuracy of the slope uncertainty prediction at facet 0 thus simply stems from the fact that the direction of the surface at this facet varies much less than at facet 0 : while the off-surface gravity uncertainties are accurately predicted, the slope predictions may not be, due to their strong non-linear dependence on the normal direction.

Another aspect of the proposed method is its ability to quantify uncertainties arising in PGM gravity fields around shape models of varying resolution. This claim is supported by 
Table 3 Facet center coordinates and associated reference slopes

\begin{tabular}{lll}
\hline & Facet 0 & Facet 1500 \\
\hline Coordinates $(\mathrm{km})$ & $(4.942 .85394 .14)^{T}$ & $(125.544-4.605-3.5761)^{T}$ \\
Slope $(\mathrm{deg})$ & 0.382 & 18.087 \\
\hline
\end{tabular}

Table 4 Comparison of the standard deviation of the slopes distribution from the

Monte-Carlo samples and those obtained from the analytical uncertainty model after folding

\begin{tabular}{lcc}
\hline Standard deviation & Facet 0 & Facet 1500 \\
\hline Monte-Carlo (deg) & 4.56 & 1.83 \\
Predicted (deg) & 4.26 & 1.86 \\
Relative error (\%) & 7.04 & -1.61 \\
\hline
\end{tabular}

an investigation of the Itokawa- 8 and Itokawa-32 shape models, respectively, comprised of 768 and 12,288 facets. The distribution of the signed vertex-to-vertex distance between these two shapes models can be crudely modeled as a zero-mean, 2.33-m standard-deviation Gaussian. The shape covariance of Itokawa- 8 capturing the surface uncertainty relative to Itokawa-32 can thus be instantiated by setting $\sigma=2.33 \mathrm{~m}$. The corresponding correlation distance $l$ should be consistent with the dimensions of the shape itself. Figure 16 highlights the two considered shape models, in addition to a comparison of the acceleration error $\delta \mathbf{a}=\mathbf{a}_{\text {Itokawa }}-\mathbf{a}_{\text {Itokawa }} 2$ with the predicted acceleration covariance $\left[P_{\mathbf{a}}\right]$ at 72 selected positions between 300 and $500 \mathrm{~m}$ away from the asteroid. This comparison metric amounts to evaluating $\sqrt{\delta \mathbf{a}^{T}\left[P_{\mathbf{a}}\right]^{-1} \delta \mathbf{a}}$ and checking whether it falls within [0,3], just like a unitvariance zero-mean Gaussian variable would. It appears that the most consistent correlation length setting is between $l=50 \mathrm{~m}$ and $l=75 \mathrm{~m}$, as this correlation distance interval yields consistent acceleration uncertainties reasonably well distributed within the 3-sigma interval. Smaller values of $l$ causes $\left[P_{\mathbf{C C}}\right]$ to mis-capture the correlated errors across the shape, producing overconfident $\left[P_{\mathbf{a}}\right]$ 's, while values of $l$ that are too large tend to correlate areas that are too far apart, yielding spuriously large values of $\left[P_{\mathbf{a}}\right]$. In any case, it is certain that parametrizing $\left[P_{\mathbf{C C}}\right]$ with only two parameters $(\sigma$ and $l)$ is an oversimplification, as more parameters would be needed to capture the areas that suffer the most from the reduced resolution. In any case, our approach makes the use of lower-resolution shape models suitable, by combining it with an uncertainty model capturing the error in the corresponding gravity field relative to a higher-resolution shape model that would be otherwise too expensive to use.

Finally, Fig. 17 depicts a side-by-side comparison of the surface slopes along with their corresponding uncertainty measure over the surface of Psyche. Although polar regions are not necessarily well captured by the proposed linearized uncertainty model, these maps are nonetheless providing valuable insight into the surface geophysical environment of imperfectly known small bodies: they demonstrate that significant uncertainty in the shape near the poles lead to non-negligible slopes uncertainties around the equator, thus indicating that preliminary shapes denoting near-zero slopes should be regarded with appropriate caution.

\subsubsection{Performance}

Some insight into the performance of the linearized gravity uncertainty model can be inferred from the respective runtimes of the uncertainty grid evaluation and that of the Monte-Carlo 

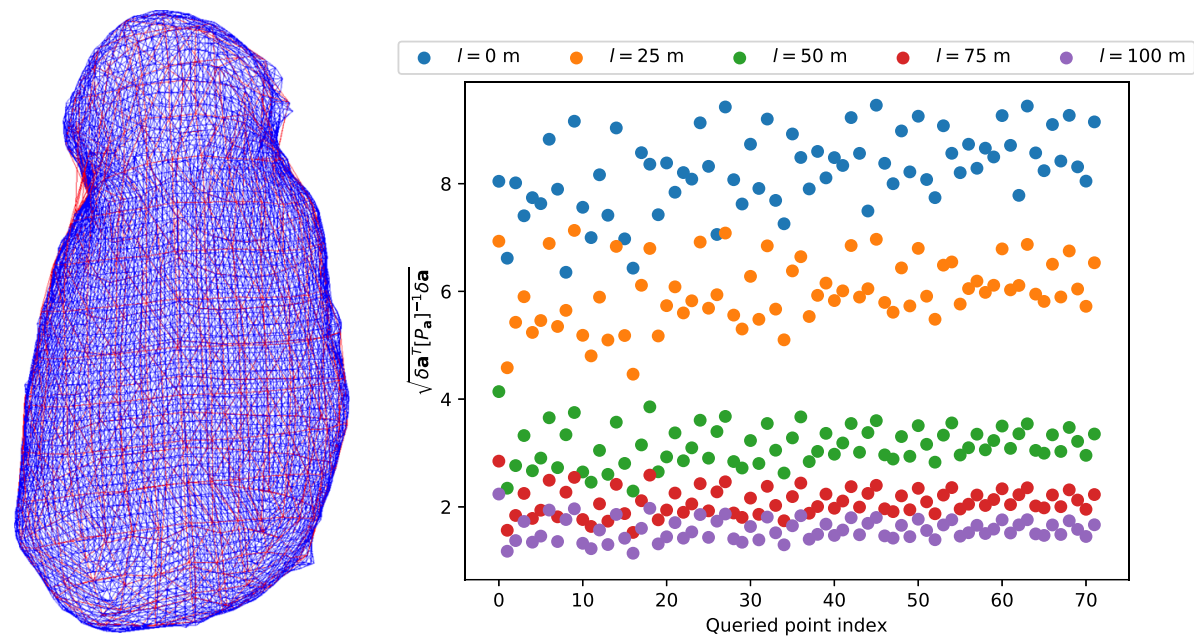

Fig. 16 Left: 25143 Itokawa-8 (red) and Itokawa-32 (blue) meshes. Right: Effect of correlation length changes on acceleration uncertainty prediction and gravity uncertainty quantification

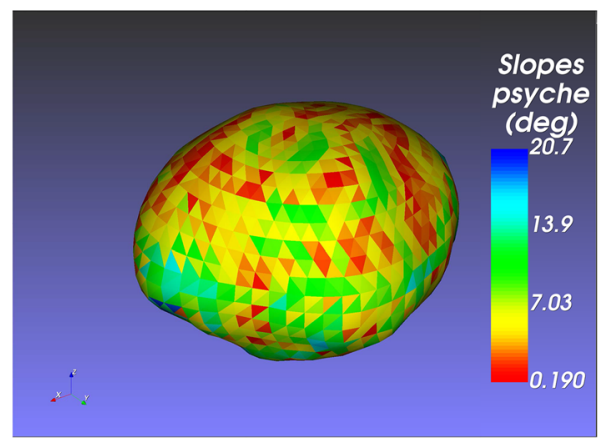

(a) Slopes (deg)

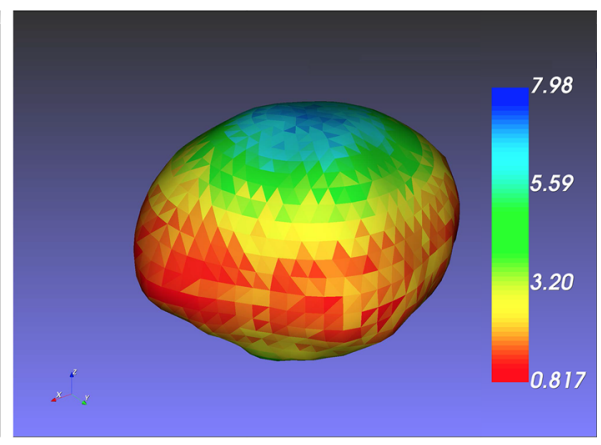

(b) Slope uncertainties (deg)

Fig. 17 Side-by-side comparison of surface slopes (left) and associated analytical one-sigma standard deviations (right) over 16 Psyche

sampling of the selected points in the gravity field validation. For instance, the simulation that was run to produce Fig. 14 featured an X-Y grid comprised of 4761 points. Evaluating the analytical prediction of the acceleration covariance over each of these points took a total of $644.3 \mathrm{~s}$ on the designated simulation computer. On the other hand, sampling the acceleration over the 5000 different shape models at each of the 48 selected points on Fig. 13 took $64.2 \mathrm{~s}$. Extrapolating the Monte-Carlo runtime to the entire grid yields a rough estimate of $6363.3 \mathrm{~s}$. For this given number of Monte-Carlo samples, the analytical gravity uncertainty quantification model is thus roughly 10 times faster than the Monte-Carlo.

\section{Conclusion}

This paper derives the expressions of the partial derivatives in the potential, acceleration and slopes computed from the Polyhedron Gravity Model with respect to the vertices of 
the underlying body. Analytical predictions of the uncertainty in the potential, acceleration and surface slopes at arbitrary locations can then be obtained in a systematic, samplingfree manner, at a lesser computational cost than Monte-Carlo sampling of the underlying shape. Besides benefitting planetary scientists and mission designers seeking for more insight into the dynamical environment of uncertain small bodies, the proposed method could be combined with a dynamic uncertainty propagation scheme allowing one to better keep track of the uncertainty in the state of an object as it evolves in the vicinity of the considered body.

\section{Code availability}

The methods developed in this paper are available within the Small Body Geophysical Analysis Tool (SBGAT) developed by Benjamin Bercovici and Jay McMahon and publicly available on GitHub (https://github.com/bbercovici/SBGAT)

Acknowledgements Benjamin Bercovici and Jay McMahon would like to express their thanks to NASA for funding this work under the grant PDART NNX16AG50G. Paolo Panicucci would like to express his gratitude to CNES for supporting him under the contract CNES 2879, and to the Fondation ISAE-SUPAERO and the ISAE SUPAERO for funding this research.

\section{Compliance with ethical standards}

Conflict of interest The authors declare that they have no conflict of interest.

\section{References}

Anthony, R.: Dobrovolskis. Inertia of any polyhedron. Icarus 124, 698-704 (1996). https://doi.org/10.1006/ icar.1996.0243. ISSN 00191035

Bercovici, B., Mcmahon, J.W.: Inertia parameter statistics of an uncertain small body shape. Icarus 328(8), 32-44 (2019). https://doi.org/10.1016/j.icarus.2019.02.016

Broschart, S., Bhaskaran, S., Bellerose, J., Dietrich, A., Han, D., HAw, R., Mastrodemos, N., Owen, W., RUsh, B., Surovik, D.: Shadow navigation support at JPL for the Rosetta landing on comet 67P. In: 26th International Symposium on Space Flight Dynamics, Number ISSFD-2017-096, Matsuyama (2017)

Busch, M.W., Ostro, S.J., Benner, L.A.M., Giorgini, J.D., Jurgens, R.F., Rose, R., Magri, C., Pravec, P., Scheeres, D.J., Broschart, S.B.: Radar and optical observations and physical modeling of near-Earth Asteroid 10115 (1992 SK). Icarus 181(1), 145-155 (2006). https://doi.org/10.1016/j.icarus.2005.10. 024. ISSN 00191035

Busch, M.W., Ostro, S.J., Benner, L.A.M., Brozovic, M., Giorgini, J.D., Jao, J.S., Scheeres, D.J., Magri, C., Nolan, M.C., Howell, E.S., Taylor, P.A., Margot, J., Brisken, W.: Radar observations and the shape of near-Earth asteroid 2008 EV5. Icarus 212(2), 649-660 (2011). https://doi.org/10.1016/j.icarus.2011.01. 013. ISSN 0019-1035

Gaskell, R., Saito, J., Ishiguro, M., Kubota, T., Hashimoto, T., Hirata, N., Abe, S., Barnouin, O.S., Scheeres, D.J.: Gaskell Itokawa shape, Model V1.0. HAY-A-AMICA-5-ITOKAWASHAPE-V1.0. NASA Planetary Data System (2008)

Groussin, O., Jorda, L., Auger, A.-T., Kührt, E., Gaskell, R., Capanna, C., Scholten, F., Preusker, F., Lamy, P., Hviid, S., et al.: Gravitational slopes, geomorphology, and material strengths of the nucleus of comet 67P/Churyumov-Gerasimenko from OSIRIS observations. Astron. Astrophys. 583, A32 (2015)

Melman, J.C.P., Mooij, E., Noomen, R.: State propagation in an uncertain asteroid gravity field. Acta Astronaut. 91, 8-19 (2013). https://doi.org/10.1016/j.actaastro.2013.04.027

Muinonen, K.: Introducing the Gaussian shape hypothesis for asteroids and comets. Astron. Astrophys. 332, 1087-1098 (1998). ISSN 00046361

Nolan, M.C., Magri, C., Howell, E.S., Benner, L.A.M., Giorgini, J.D., Hergenrother, C.W., Hudson, R.S., Lauretta, D., Margot, J.-L., Ostro, S.J., Scheeres, D.J.: Shape model and surface properties of the OSIRIS- 
REx target Asteroid (101955) Bennu from radar and lightcurve observations. Icarus 226(1), 629-640 (2013). https://doi.org/10.1016/j.icarus.2013.05.028. ISSN 00191035

Oguri, K., Lantoine, G., Hart, B., McMahon, J.W.: Science orbit design with frozen beta angle: theory and application to psyche mission. In: AAS/AIAA Space Flight Mechanics Meeting, pp. 1-15, Ka'anapali, HI (2019)

Oh, D.Y., Collins, S., Goebel, D., Hart, B., Lantoine, G., Snyder, S., Whiffen, G., Elkins-tanton, L., Lord, P., Pirkl, Z., Rotlisburger, L.: Development of the psyche mission for NASA's discovery program. In: 35th International Electric Propulsion Conference, pp. 1-19 (2017)

O'Keefe, S.A., Schaub, H.: Shadow set considerations for modified rodrigues parameter attitude filtering. Adv. Astronaut. Sci. 150(6), 2777-2786 (2014). https://doi.org/10.2514/1.G000405. ISSN 00653438

Ostro, S.J., Hudson, R.S., Benner, L.A.M., Giorgini, J.D., Magri, C., Margot, J.-L., Nolan, M.C.: Asteroid radar astronomy. Asteroids III, pp. 151-168 (2002)

Owen, W.M., Wang. T.C.: NEAR optical navigation at Eros. In: AAS/AIAA Astrodynamics Specialist Conference, Quebec City, Canada (2001)

Panicucci, P., Bercovici, B., Zenou, E., McMahon, J.W., Delpech, M., Lebreton, J., Kanani, K.: Uncertainties in the gravity spherical harmonics coefficients arising from a stochastic polyhedral shape. Celest. Mech. Dyn. Astron. (2020). https://doi.org/10.1007/s10569-020-09962-8. ISSN 1572-9478

Pesce, V., Agha-mohammadi, A.-A., Lavagna, M.: Autonomous navigation and mapping of small bodies. In: 2018 IEEE Aerospace Conference (2018). https://doi.org/10.1109/AERO.2018.8396797

Ren, Y., Shan, J.: Reliability-based soft landing trajectory optimization near asteroid with uncertain gravitational field. J. Guid. Control Dyn. (2015). https://doi.org/10.2514/1.G000903

Scheeres, D.J., Fahnestock, E.G., Ostro, S.J., Margot, J.-L., Benner, L.A.M., Broschart, S., Bellerose, J., Giorgini, J.D., Nolan, M.C., Magri, C., Pravec, P., Scheirich, P., Rose, R., Jurgens, R.F., De Jong, E.M., Suzuki, S., De Jong, E.M.: Dynamical configuration of binary Near-Earth Asteroid (66391) 1999 KW4. Science 314, 1280-1283 (2006a). https://doi.org/10.1126/science.1133622. ISSN 0036-8075

Scheeres, D.J., Gaskell, R.W., Abe, S., Barnouin, O.S., Hashimoto, T., Kawaguchi, J., Kubota, T., Saito, J., Yoshikawa, M., Hirata, N., Mukai, T., Ishiguro, M., Kominato, T., Shirakawa, K., Uo, M.: The actual dynamical environment about Itokawa. In: AIAA/AAS Astrodynamics Specialist Conference and Exhibit, number August, (2006b). https://doi.org/10.2514/6.2006-6661

Scheeres, D.J., Hesar, S.G., Tardivel, S., Hirabayashi, M., Farnocchia, D., McMahon, J.W., Chesley, S.R., Barnouin, O.S., Binzel, R.P., Bottke, W.F., Daly, M.G., Emery, J.P., Hergenrother, C.W., Lauretta, D., Marshall, J.R., Michel, P., Nolan, M.C., Walsh, K.J.: The geophysical environment of Bennu. Icarus 276, 116-140 (2016). https://doi.org/10.1016/j.icarus.2016.04.013. ISSN 0019-1035

Shepard, M.K., Richardson, J., Taylor, P.A., Rodriguez-ford, L.A., Conrad, A., De Pater, I., Adamkovics, M., De Kleer, K., Males, J.R., Morzinski, K.M., Close, L.M., Kaasalainen, M., Viikinkoski, M., Timerson, B., Reddy, V., Magri, C., Nolan, M.C., Howell, E.S., Benner, L.A.M., Giorgini, J.D., Warner, B.D., Harris, A.W.: Radar observations and shape model of asteroid 16 Psyche. Icarus 281, 388-403 (2017). https:// doi.org/10.1016/j.icarus.2016.08.011. ISSN 0019-1035

Tedesco, E.F., Noah, P.V., Noah, M., Price, S.D.: The supplemental IRAS minor planet survey. Astron. J. 123(2), 1056-1085 (2002)

Torppa, J., Hentunen, V.P., Pääkkönen, P., Kehusmaa, P., Muinonen, K.: Asteroid shape and spin statistics from convex models. Icarus 198(1), 91-107 (2008). https://doi.org/10.1016/j.icarus.2008.07.014. ISSN 00191035

Torppa, J., Kaasalainen, M., Michałowski, T., Kwiatkowski, T., Kryszczyńska, A., Denchev, P., Kowalski, R.: Shapes and rotational properties of thirty asteroids from photometric data. Icarus 164(2), 346-383 (2003). https://doi.org/10.1016/S0019-1035(03)00146-5. ISSN 00191035

Tsoulis, D., Petrovic, S.: On the singularities of the gravity field of a homogeneous polyhedral body. Geophysics 66(2), 535-539 (2001)

Werner, R.A.: Spherical harmonic coefficients for the potential of a constant-density polyhedron. Comput. Geosci. 23(10), 1071-1077 (1997). https://doi.org/10.1016/S0098-3004(97)00110-6. ISSN 00983004

Werner, R.A., Scheeres, D.J.: Exterior gravitation of a polyhedron derived and compared with harmonic and mascon gravitation representations of asteroid 4769 Castalia. Celest. Mech. Dyn. Astron. 65(3), 313-344 (1997). https://doi.org/10.1007/BF00053511. ISSN 0923-2958

Williams, B.G., Antreasian, P.G., Bordi, J.J., Carranza, E., Chesley, S.R., Helfrich, C.E., Miller, J.K., Owen, W.M., Wang, T.C.: Navigation for NEAR Shoemaker: the first spacecraft to Orbit an Asteroid. In: AAS/AIAA Astrodynamics Specialist Conference (2001) 